\title{
Higher Sugawara operators for the quantum affine algebras of type $A$
}

\author{
Luc Frappat, Naihuan Jing, Alexander Molev and Eric Ragoucy
}

\begin{abstract}
We give explicit formulas for the elements of the center of the completed quantum affine algebra in type $A$ at the critical level which are associated with the fundamental representations. We calculate the images of these elements under a Harish-Chandratype homomorphism. These images coincide with those in the free field realization of the quantum affine algebra and reproduce generators of the $q$-deformed classical $\mathcal{W}$-algebra of Frenkel and Reshetikhin.
\end{abstract}

Preprint LAPTH-027/15

\section{Introduction}

Let $\mathfrak{g}$ be a simple Lie algebra over $\mathbb{C}$ and let $\mathrm{U}_{q}(\widehat{\mathfrak{g}})$ denote the quantum affine algebra associated with $\mathfrak{g}$. Due to the work of Reshetikhin and Semenov-Tian-Shansky [27], to every finite-dimensional representation $V$ of $\mathrm{U}_{q}(\widehat{\mathfrak{g}})$ one can associate a formal Laurent series $\ell_{V}(z)$ whose coefficients belong to the center $\mathrm{Z}_{q}(\widehat{\mathfrak{g}})$ of the completion $\widetilde{\mathrm{U}}_{q}(\widehat{\mathfrak{g}})_{\text {cri }}$ of the quantum affine algebra at the critical level. The map $V \mapsto \ell_{V}(z)$ was further studied by Ding and Etingof [9] who showed that if the coefficients of $\ell_{V}(z)$ are regarded as operators on highest weight modules at the critical level, then it possesses properties of a homomorphism from the Grothendieck ring $\operatorname{Rep} \mathrm{U}_{q}(\widehat{\mathfrak{g}})$ to formal series in $z$. Furthermore, the coefficients of $\ell_{V}(z)$ were shown to generate all singular vectors in Verma modules [9]. This relied upon connections of the series $\ell_{V}(z)$ with transfer matrices; see also work of Frenkel and Reshetikhin [18, Sec. 8] for its relationship with the $q$-characters of finite-dimensional representations of $\mathrm{U}_{q}(\widehat{\mathfrak{g}})$.

By a conjecture of Frenkel and Reshetikhin [17], [18, the center $\mathrm{Z}_{q}(\widehat{\mathfrak{g}})$ is isomorphic to the $q$-deformed classical $\mathcal{W}$-algebra, as a Poisson algebra. More precisely, Conjecture 1 of [17] applies to the completion of the central subalgebra of $\widetilde{\mathrm{U}}_{q}(\widehat{\mathfrak{g}})_{\text {cri }}$ generated by the 
coefficients of the series $\ell_{V_{1}}(z), \ldots, \ell_{V_{n}}(z)$ associated with all fundamental representations $V_{i}$ of $\mathrm{U}_{q}(\widehat{\mathfrak{g}})$. Its proof was sketched in [17] for $\mathfrak{g}=\mathfrak{s l}_{N}$. The isomorphism is provided by the free field (or Wakimoto) realization of the quantum affine algebra due to Awata, Odake and Shiraishi [1] which extended an earlier work [19] on the vertex representations from level 1 to an arbitrary level.

The results of [17] generalize the Feigin-Frenkel theorem which establishes a Poisson algebra isomorphism between the center $\mathfrak{z}(\widehat{\mathfrak{g}})$ of the affine vertex algebra $V(\mathfrak{g})$ at the critical level and the classical $\mathcal{W}$-algebra associated with the Langlands dual Lie algebra ${ }^{L} \mathfrak{g}$; see [15] for a detailed exposition. The Feigin-Frenkel center $\mathfrak{z}(\widehat{\mathfrak{g}})$ is an algebra of polynomials which can be identified with a commutative subalgebra of the universal enveloping algebra $\mathrm{U}\left(t^{-1} \mathfrak{g}\left[t^{-1}\right]\right)$. As discovered by Feigin, Frenkel and Reshetikhin [13, the higher degree Hamiltonians in the Gaudin model can be obtained from generators of $\mathfrak{z}(\widehat{\mathfrak{g}})$; see also [14] and [28]. Explicit constructions of generators of $\mathfrak{z}(\widehat{\mathfrak{g}})$ were given in [7], [8] and [24] for types $A, B, C$ and $D$; see also [25] for their images in the classical $\mathcal{W}$-algebras and [26] for super-analogues of these constructions with $\mathfrak{g}=\mathfrak{g l}_{m \mid n}$.

Our goal in this paper is to give similar explicit formulas for higher degree Sugawara operators for $\mathrm{U}_{q}\left(\widehat{\mathfrak{g l}}_{n}\right)$; i.e., for elements of the center of $\widetilde{\mathrm{U}}_{q}\left(\widehat{\mathfrak{g l}}_{n}\right)_{\text {cri }}$ (Theorem 3.2). The formulas express the operators in terms of the $R L L$-presentation $\mathrm{U}_{q}\left(\widehat{\mathfrak{g l}}_{n}\right)$; see [20] and [27]. We use a version of the Poincaré-Birkhoff-Witt theorem for this presentation to introduce an analogue of the Harish-Chandra homomorphism and calculate the images of the central elements under the homomorphism.

Then we apply the Ding-Frenkel isomorphism [10] between the $R L L$ and Drinfeld presentations to calculate the images of the Sugawara operators in the $q$-deformed classical $\mathcal{W}$-algebra by using the approach of [17] based on the free field realization [1]. Our generators correspond to the fundamental representations of $\mathrm{U}_{q}\left(\widehat{\mathfrak{g l}}_{n}\right)$ and essentially coincide with those of [17] up to an appropriate identification of the parameters. The construction involves a fusion formula for the $q$-deformed antisymmetrizer expressing it in terms of the trigonometric $R$-matrices.

As an application of Theorem 3.2, we produce explicit invariants of the $q$-analogue $V_{q}\left(\mathfrak{g l}_{n}\right)$ of the vacuum module over the quantum affine algebra. The invariants are obtained by the action of the higher degree Sugawara operators on the vacuum vector; cf. [18].

\section{Quantum affine algebra}

We use the $R L L$ presentation of $\mathrm{U}_{q}\left(\widehat{\mathfrak{g l}}_{n}\right)$ introduced in [27]; see also [20]. We regard $q$ as a nonzero complex number which is not a root of unity. Introduce the two-parameter 
$R$-matrix $R(u, v) \in$ End $\mathbb{C}^{n} \otimes$ End $\mathbb{C}^{n}$ by

$$
\begin{aligned}
R(u, v) & =(u-v) \sum_{i \neq j} e_{i i} \otimes e_{j j}+\left(q^{-1} u-q v\right) \sum_{i} e_{i i} \otimes e_{i i} \\
& +\left(q^{-1}-q\right) u \sum_{i>j} e_{i j} \otimes e_{j i}+\left(q^{-1}-q\right) v \sum_{i<j} e_{i j} \otimes e_{j i},
\end{aligned}
$$

where $e_{i j} \in$ End $\mathbb{C}^{n}$ are the standard matrix units. We will also need the one-parameter $R$-matrices

$$
\begin{aligned}
\bar{R}(x)=\frac{R(x, 1)}{q^{-1} x-q} & =\sum_{i} e_{i i} \otimes e_{i i}+\frac{1-x}{q-q^{-1} x} \sum_{i \neq j} e_{i i} \otimes e_{j j} \\
& +\frac{\left(q-q^{-1}\right) x}{q-q^{-1} x} \sum_{i>j} e_{i j} \otimes e_{j i}+\frac{q-q^{-1}}{q-q^{-1} x} \sum_{i<j} e_{i j} \otimes e_{j i}
\end{aligned}
$$

and

$$
R(x)=f(x) \bar{R}(x)
$$

where

$$
f(x)=1+\sum_{k=1}^{\infty} f_{k} x^{k}, \quad f_{k}=f_{k}(q),
$$

is a formal power series in $x$ whose coefficients $f_{k}$ are rational functions in $q$ uniquely determined by the relation

$$
f\left(x q^{2 n}\right)=f(x) \frac{\left(1-x q^{2}\right)\left(1-x q^{2 n-2}\right)}{(1-x)\left(1-x q^{2 n}\right)} .
$$

They can be found by the recurrence

$$
f_{k}=-\frac{\left(1-q^{2}\right)\left(1-q^{2 n-2}\right)}{1-q^{2 n}} \sum_{i=1}^{k} \frac{1-q^{2 n i}}{1-q^{2 n k}} f_{k-i}, \quad k \geqslant 1,
$$

with $f_{0}=1$. Equivalently, $f(x)$ can be given by

$$
f(x)=\frac{\left(x ; q^{2 n}\right)_{\infty}\left(x q^{2 n} ; q^{2 n}\right)_{\infty}}{\left(x q^{2} ; q^{2 n}\right)_{\infty}\left(x q^{2 n-2} ; q^{2 n}\right)_{\infty}}, \quad(a ; b)_{\infty}:=\prod_{r=0}^{\infty}\left(1-a b^{r}\right),
$$

where the coefficients of the powers $x^{k}$ are power series in $q$ converging to $f_{k}$ for $|q|<1$.

The quantum affine algebra $\mathrm{U}_{q}\left(\widehat{\mathfrak{g l}}_{n}\right)$ is generated by elements

$$
l_{i j}^{+}[-r], \quad l_{i j}^{-}[r] \quad \text { with } \quad 1 \leqslant i, j \leqslant n, \quad r=0,1, \ldots,
$$


and the invertible central element $q^{c}$, subject to the defining relations

$$
\begin{aligned}
& l_{j i}^{+}[0]=l_{i j}^{-}[0]=0 \quad \text { for } \quad 1 \leqslant i<j \leqslant n, \\
& l_{i i}^{+}[0] l_{i i}^{-}[0]=l_{i i}^{-}[0] l_{i i}^{+}[0]=1 \quad \text { for } \quad i=1, \ldots, n,
\end{aligned}
$$

and

$$
\begin{aligned}
R(u / v) L_{1}^{ \pm}(u) L_{2}^{ \pm}(v) & =L_{2}^{ \pm}(v) L_{1}^{ \pm}(u) R(u / v), \\
R\left(u q^{-c} / v\right) L_{1}^{+}(u) L_{2}^{-}(v) & =L_{2}^{-}(v) L_{1}^{+}(u) R\left(u q^{c} / v\right) .
\end{aligned}
$$

In the last two relations we consider the matrices $L^{ \pm}(u)=\left[l_{i j}^{ \pm}(u)\right]$, whose entries are formal power series in $u$ and $u^{-1}$,

$$
l_{i j}^{+}(u)=\sum_{r=0}^{\infty} l_{i j}^{+}[-r] u^{r}, \quad l_{i j}^{-}(u)=\sum_{r=0}^{\infty} l_{i j}^{-}[r] u^{-r} .
$$

Here and below we regard the matrices as elements

$$
L^{ \pm}(u)=\sum_{i, j=1}^{n} e_{i j} \otimes l_{i j}^{ \pm}(u) \in \text { End } \mathbb{C}^{n} \otimes \mathrm{U}_{q}\left(\widehat{\mathfrak{g l}}_{n}\right)\left[\left[u^{ \pm 1}\right]\right]
$$

and use a subscript to indicate a copy of the matrix in the multiple tensor product algebra

$$
\underbrace{\text { End } \mathbb{C}^{n} \otimes \ldots \otimes \operatorname{End} \mathbb{C}^{n}}_{k} \otimes \mathrm{U}_{q}\left(\widehat{\mathfrak{g l}}_{n}\right)\left[\left[u^{ \pm 1}\right]\right]
$$

so that

$$
L_{a}^{ \pm}(u)=\sum_{i, j=1}^{n} 1^{\otimes(a-1)} \otimes e_{i j} \otimes 1^{\otimes(k-a)} \otimes l_{i j}^{ \pm}(u) .
$$

In particular, we take $k=2$ for the defining relations (2.7) and (2.8).

This notation for elements of algebras of the form (2.10) will be extended as follows. For an element

$$
C=\sum_{i, j, r, s=1}^{n} c_{i j r s} e_{i j} \otimes e_{r s} \in \operatorname{End} \mathbb{C}^{n} \otimes \operatorname{End} \mathbb{C}^{n},
$$

and any two indices $a, b \in\{1, \ldots, k\}$ such that $a \neq b$, we denote by $C_{a b}$ the element of the algebra $\left(\text { End } \mathbb{C}^{n}\right)^{\otimes k}$ with $k \geqslant 2$ given by

$$
C_{a b}=\sum_{i, j, r, s=1}^{n} c_{i j r s}\left(e_{i j}\right)_{a}\left(e_{r s}\right)_{b}, \quad\left(e_{i j}\right)_{a}=1^{\otimes(a-1)} \otimes e_{i j} \otimes 1^{\otimes(k-a)} .
$$

We regard the matrix transposition as the linear map

$$
t: \text { End } \mathbb{C}^{n} \rightarrow \text { End } \mathbb{C}^{n}, \quad e_{i j} \mapsto e_{j i} .
$$


For any $a \in\{1, \ldots, k\}$ we will denote by $t_{a}$ the corresponding partial transposition on the algebra (2.10) which acts as $t$ on the $a$-th copy of End $\mathbb{C}^{n}$ and as the identity map on all the other tensor factors.

The $R$-matrix (2.3) satisfies the following crossing symmetry relations [20]:

$$
\left(R_{12}(x)^{-1}\right)^{t_{2}} D_{2} R_{12}\left(x q^{2 n}\right)^{t_{2}}=D_{2} \quad \text { and } \quad R_{12}\left(x q^{2 n}\right)^{t_{1}} D_{1}\left(R_{12}(x)^{-1}\right)^{t_{1}}=D_{1},
$$

where $D$ denotes the diagonal $n \times n$ matrix

$$
D=\operatorname{diag}\left[q^{n-1}, q^{n-3}, \ldots, q^{-n+1}\right]
$$

with the meaning of subscripts as in (2.12).

\section{Main theorem}

Denote by $\mathrm{U}_{q}\left(\widehat{\mathfrak{g l}}_{n}\right)_{\text {cri }}$ the quantum affine algebra at the critical level $c=-n$, which is the quotient of $\mathrm{U}_{q}\left(\widehat{\mathfrak{g l}}_{n}\right)$ by the relation $q^{c}=q^{-n}$. Its completion $\widetilde{\mathrm{U}}_{q}\left(\widehat{\mathfrak{g l}}_{n}\right)_{\text {cri }}$ is defined as the inverse limit

$$
\widetilde{\mathrm{U}}_{q}\left(\widehat{\mathfrak{g l}}_{n}\right)_{\mathrm{cri}}=\lim _{\longleftarrow} \mathrm{U}_{q}\left(\widehat{\mathfrak{g l}}_{n}\right)_{\text {cri }} / J_{p}, \quad p>0,
$$

where $J_{p}$ denotes the left ideal of $\mathrm{U}_{q}\left(\widehat{\mathfrak{g l}}_{n}\right)_{\text {cri }}$ generated by all elements $l_{i j}^{-}[r]$ with $r \geqslant p$. Elements of the center $\mathrm{Z}_{q}\left(\widehat{\mathfrak{g l}}_{n}\right)$ of $\widetilde{\mathrm{U}}_{q}\left(\widehat{\mathfrak{g l}}_{n}\right)_{\text {cri }}$ are known as Sugawara operators.

Consider the $q$-permutation operator $P^{q} \in \operatorname{End}\left(\mathbb{C}^{n} \otimes \mathbb{C}^{n}\right) \cong$ End $\mathbb{C}^{n} \otimes$ End $\mathbb{C}^{n}$ defined by

$$
P^{q}=\sum_{i} e_{i i} \otimes e_{i i}+q \sum_{i>j} e_{i j} \otimes e_{j i}+q^{-1} \sum_{i<j} e_{i j} \otimes e_{j i} .
$$

The symmetric group $\mathfrak{S}_{k}$ acts on the space $\left(\mathbb{C}^{n}\right)^{\otimes k}$ by $s_{i} \mapsto P_{s_{i}}^{q}:=P_{i, i+1}^{q}$ for $i=1, \ldots, k-1$, where $s_{i}$ denotes the transposition $(i, i+1)$. If $\sigma=s_{i_{1}} \cdots s_{i_{l}}$ is a reduced decomposition of an element $\sigma \in \mathfrak{S}_{k}$ we set $P_{\sigma}^{q}=P_{s_{i_{1}}}^{q} \cdots P_{s_{i_{l}}}^{q}$. We denote by $A^{(k)}$ the image of the normalized antisymmetrizer associated with the $q$-permutations:

$$
A^{(k)}=\frac{1}{k !} \sum_{\sigma \in \mathfrak{S}_{k}} \operatorname{sgn} \sigma \cdot P_{\sigma}^{q}
$$

so that $\left(A^{(k)}\right)^{2}=A^{(k)}$.

For each $k=1, \ldots, n$ introduce the Laurent series $\ell_{k}(z)$ in $z$ by

$$
\ell_{k}(z)=\operatorname{tr}_{1, \ldots, k} A^{(k)} L_{1}^{+}(z) \ldots L_{k}^{+}\left(z q^{-2 k+2}\right) L_{k}^{-}\left(z q^{-n-2 k+2}\right)^{-1} \ldots L_{1}^{-}\left(z q^{-n}\right)^{-1} D_{1} \ldots D_{k},
$$

where $D$ is the diagonal matrix (2.14) and the trace is taken over all $k$ copies of End $\mathbb{C}^{n}$ in (2.10). All coefficients of the series $\ell_{k}(z)$ are elements of the algebra $\widetilde{U}_{q}\left(\widehat{\mathfrak{g l}}_{n}\right)_{\text {cri }}$. We will also need an equivalent formula for $\ell_{k}(z)$ which is obtained by using the following well-known particular case of the fusion procedure for the $R$-matrix (2.1); see [5]. 
Lemma 3.1. Set $v_{a}=z q^{-2 a+2}$ for $a=1, \ldots, k$. We have

$$
\prod_{1 \leqslant a<b \leqslant k} R_{a b}\left(v_{a}, v_{b}\right)=k ! z^{k(k-1) / 2} \prod_{0 \leqslant a<b \leqslant k-1}\left(q^{-2 a}-q^{-2 b}\right) A^{(k)},
$$

where the product is taken in the lexicographical order on the pairs $(a, b)$.

Applying (2.7) and Lemma 3.1 we obtain the relations

$$
\begin{aligned}
A^{(k)} L_{1}^{+}\left(v_{1}\right) \ldots L_{k}^{+}\left(v_{k}\right) & =L_{k}^{+}\left(v_{k}\right) \ldots L_{1}^{+}\left(v_{1}\right) A^{(k)} \\
A^{(k)} L_{k}^{-}\left(v_{k} q^{-n}\right)^{-1} \ldots L_{1}^{-}\left(v_{1} q^{-n}\right)^{-1} & =L_{1}^{-}\left(v_{1} q^{-n}\right)^{-1} \ldots L_{k}^{-}\left(v_{k} q^{-n}\right)^{-1} A^{(k)} .
\end{aligned}
$$

We also have

$$
R(u, v) D_{1} D_{2}=D_{2} D_{1} R(u, v)
$$

and $D_{1} D_{2}=D_{2} D_{1}$ so that

$$
A^{(k)} D_{1} \ldots D_{k}=D_{1} \ldots D_{k} A^{(k)}
$$

Thus, $\ell_{k}(z)$ defined in (3.4) can also be given by the formula

$$
\ell_{k}(z)=\operatorname{tr}_{1, \ldots, k} L_{k}^{+}\left(v_{k}\right) \ldots L_{1}^{+}\left(v_{1}\right) L_{1}^{-}\left(v_{1} q^{-n}\right)^{-1} \ldots L_{k}^{-}\left(v_{k} q^{-n}\right)^{-1} D_{1} \ldots D_{k} A^{(k)} .
$$

The following is our main result which provides explicit formulas for higher Sugawara operators.

Theorem 3.2. The coefficients of $\ell_{k}(z)$ belong to the center of the completed quantum affine algebra at the critical level $\widetilde{\mathrm{U}}_{q}\left(\widehat{\mathfrak{g l}}_{n}\right)_{\text {cri }}$ for all $k=1, \ldots, n$.

Proof. Introduce an extra copy of the endomorphism algebra End $\mathbb{C}^{n}$ in (2.10) and label it by 0 to work with the algebra

$$
\text { End } \mathbb{C}^{n} \otimes\left(\text { End } \mathbb{C}^{n}\right)^{\otimes k} \otimes \widetilde{\mathrm{U}}_{q}\left(\widehat{\mathfrak{g l}}_{n}\right)_{\mathrm{cri}}
$$

It will be sufficient to verify what $\ell_{k}(z)$ commutes with $L_{0}^{ \pm}(u)$. By using (2.7) we get

$$
\begin{aligned}
L_{0}^{+}(u) & L_{k}^{+}\left(v_{k}\right) \ldots L_{1}^{+}\left(v_{1}\right) \\
& =R_{0 k}\left(u / v_{k}\right)^{-1} \ldots R_{01}\left(u / v_{1}\right)^{-1} R_{01}\left(u / v_{1}\right) \ldots R_{0 k}\left(u / v_{k}\right) L_{0}^{+}(u) L_{k}^{+}\left(v_{k}\right) \ldots L_{1}^{+}\left(v_{1}\right) \\
& =R_{0 k}\left(u / v_{k}\right)^{-1} \ldots R_{01}\left(u / v_{1}\right)^{-1} L_{k}^{+}\left(v_{k}\right) \ldots L_{1}^{+}\left(v_{1}\right) L_{0}^{+}(u) R_{01}\left(u / v_{1}\right) \ldots R_{0 k}\left(u / v_{k}\right) .
\end{aligned}
$$

Relation (2.8) implies

$$
L_{0}^{+}(u) R_{0 a}\left(u / v_{a}\right) L_{a}^{-}\left(v_{a} q^{-n}\right)^{-1}=L_{a}^{-}\left(v_{a} q^{-n}\right)^{-1} R_{0 a}\left(u q^{2 n} / v_{a}\right) L_{0}^{+}(u), \quad a=1, \ldots, k,
$$


and so

$$
\begin{aligned}
L_{0}^{+}(u) R_{01}\left(u / v_{1}\right) \ldots & R_{0 k}\left(u / v_{k}\right) L_{1}^{-}\left(v_{1} q^{-n}\right)^{-1} \ldots L_{k}^{-}\left(v_{k} q^{-n}\right)^{-1} \\
& =L_{1}^{-}\left(v_{1} q^{-n}\right)^{-1} \ldots L_{k}^{-}\left(v_{k} q^{-n}\right)^{-1} R_{01}\left(u q^{2 n} / v_{1}\right) \ldots R_{0 k}\left(u q^{2 n} / v_{k}\right) L_{0}^{+}(u) .
\end{aligned}
$$

Thus, to conclude that $L_{0}^{+}(u) \ell_{k}(z)=\ell_{k}(z) L_{0}^{+}(u)$ we need to show that the trace

$$
\begin{aligned}
\operatorname{tr}_{1, \ldots, k} & R_{0 k}\left(u / v_{k}\right)^{-1} \ldots R_{01}\left(u / v_{1}\right)^{-1} L_{k}^{+}\left(v_{k}\right) \ldots L_{1}^{+}\left(v_{1}\right) \\
& \times L_{1}^{-}\left(v_{1} q^{-n}\right)^{-1} \ldots L_{k}^{-}\left(v_{k} q^{-n}\right)^{-1} R_{01}\left(u q^{2 n} / v_{1}\right) \ldots R_{0 k}\left(u q^{2 n} / v_{k}\right) D_{1} \ldots D_{k} A^{(k)}
\end{aligned}
$$

equals $\ell_{k}(z)$. The $R$-matrix $R(u, v)$ satisfies the Yang-Baxter equation

$$
R_{12}(u, v) R_{13}(u, w) R_{23}(v, w)=R_{23}(v, w) R_{13}(u, w) R_{12}(u, v)
$$

which implies

$$
R_{12}(u, v) R_{13}(u / w) R_{23}(v / w)=R_{23}(v / w) R_{13}(u / w) R_{12}(u, v) .
$$

Therefore, Lemma 3.1 gives

$$
\begin{gathered}
R_{01}\left(u q^{2 n} / v_{1}\right) \ldots R_{0 k}\left(u q^{2 n} / v_{k}\right) A^{(k)}=A^{(k)} R_{0 k}\left(u q^{2 n} / v_{k}\right) \ldots R_{01}\left(u q^{2 n} / v_{1}\right), \\
R_{0 k}\left(u / v_{k}\right)^{-1} \ldots R_{01}\left(u / v_{1}\right)^{-1} A^{(k)}=A^{(k)} R_{01}\left(u / v_{1}\right)^{-1} \ldots R_{0 k}\left(u / v_{k}\right)^{-1} .
\end{gathered}
$$

Applying (3.5), (3.6), (3.7) and (3.11) to the expression under the trace in (3.10), we will bring it to the form

$$
\begin{aligned}
\operatorname{tr}_{1, \ldots, k} R_{0 k} & \left(u / v_{k}\right)^{-1} \ldots R_{01}\left(u / v_{1}\right)^{-1} A^{(k)} L_{1}^{+}\left(v_{1}\right) \ldots L_{k}^{+}\left(v_{k}\right) \\
& \times L_{k}^{-}\left(v_{k} q^{-n}\right)^{-1} \ldots L_{1}^{-}\left(v_{1} q^{-n}\right)^{-1} R_{0 k}\left(u q^{2 n} / v_{k}\right) \ldots R_{01}\left(u q^{2 n} / v_{1}\right) D_{1} \ldots D_{k} .
\end{aligned}
$$

Now write $A^{(k)}=\left(A^{(k)}\right)^{2}$ and move one copy of $A^{(k)}$ to the left by using (3.12), and move the other copy back to its right-most position. We get

$$
\begin{aligned}
\operatorname{tr}_{1, \ldots, k} & A^{(k)} R_{01}\left(u / v_{1}\right)^{-1} \ldots R_{0 k}\left(u / v_{k}\right)^{-1} L_{k}^{+}\left(v_{k}\right) \ldots L_{1}^{+}\left(v_{1}\right) \\
& \times L_{1}^{-}\left(v_{1} q^{-n}\right)^{-1} \ldots L_{k}^{-}\left(v_{k} q^{-n}\right)^{-1} R_{01}\left(u q^{2 n} / v_{1}\right) \ldots R_{0 k}\left(u q^{2 n} / v_{k}\right) D_{1} \ldots D_{k} A^{(k)} .
\end{aligned}
$$

Use the cyclic property of trace to move the left copy of $A^{(k)}$ to the right-most position and replace $\left(A^{(k)}\right)^{2}$ with $A^{(k)}$. As a result of these transformations, the order of the first $k$ factors in (3.10) will be reversed, while the rest of the expression remains unchanged. Therefore, we can also write it in the form (3.13) with the order of the first $k$ factors reversed; that is, of the form $\operatorname{tr}_{1, \ldots, k} X Y$ with

$$
X=R_{01}\left(u / v_{1}\right)^{-1} \ldots R_{0 k}\left(u / v_{k}\right)^{-1} A^{(k)} L_{1}^{+}\left(v_{1}\right) \ldots L_{k}^{+}\left(v_{k}\right) L_{k}^{-}\left(v_{k} q^{-n}\right)^{-1} \ldots L_{1}^{-}\left(v_{1} q^{-n}\right)^{-1}
$$


and

$$
Y=R_{0 k}\left(u q^{2 n} / v_{k}\right) \ldots R_{01}\left(u q^{2 n} / v_{1}\right) D_{1} \ldots D_{k} .
$$

Now use the property

$$
\operatorname{tr}_{1, \ldots, k} X Y=\operatorname{tr}_{1, \ldots, k} X^{t_{1} \ldots t_{k}} Y^{t_{1} \ldots t_{k}}
$$

We have

$$
X^{t_{1} \ldots t_{k}}=L^{t_{1} \ldots t_{k}}\left(R_{01}\left(u / v_{1}\right)^{-1}\right)^{t_{1}} \ldots\left(R_{0 k}\left(u / v_{k}\right)^{-1}\right)^{t_{k}},
$$

where we have set

$$
L=A^{(k)} L_{1}^{+}\left(v_{1}\right) \ldots L_{k}^{+}\left(v_{k}\right) L_{k}^{-}\left(v_{k} q^{-n}\right)^{-1} \ldots L_{1}^{-}\left(v_{1} q^{-n}\right)^{-1} .
$$

Furthermore,

$$
Y^{t_{1} \ldots t_{k}}=D_{1} \ldots D_{k} R_{0 k}\left(u q^{2 n} / v_{k}\right)^{t_{k}} \ldots R_{01}\left(u q^{2 n} / v_{1}\right)^{t_{1}} .
$$

Hence, the first crossing symmetry relation in (2.13) gives

$$
\begin{aligned}
\operatorname{tr}_{1, \ldots, k} X^{t_{1} \ldots t_{k}} Y^{t_{1} \ldots t_{k}} & =\operatorname{tr}_{1, \ldots, k} L^{t_{1} \ldots t_{k}} D_{1} \ldots D_{k} \\
& =\operatorname{tr}_{1, \ldots, k} A^{(k)} L_{1}^{+}\left(v_{1}\right) \ldots L_{k}^{+}\left(v_{k}\right) L_{k}^{-}\left(v_{k} q^{-n}\right)^{-1} \ldots L_{1}^{-}\left(v_{1} q^{-n}\right)^{-1} D_{1} \ldots D_{k}
\end{aligned}
$$

which coincides with $\ell_{k}(z)$ as defined in (3.4) thus completing the proof for $L_{0}^{+}(u)$.

The argument showing that $L_{0}^{-}(u) \ell_{k}(z)=\ell_{k}(z) L_{0}^{-}(u)$ is quite similar, so we only briefly outline it. Using (2.8) we get

$$
\begin{aligned}
& L_{0}^{-}(u) L_{k}^{+}\left(v_{k}\right) \ldots L_{1}^{+}\left(v_{1}\right)=R_{k 0}\left(v_{k} q^{n} / u\right) \ldots R_{10}\left(v_{1} q^{n} / u\right) \\
& \quad \times L_{k}^{+}\left(v_{k}\right) \ldots L_{1}^{+}\left(v_{1}\right) L_{0}^{-}(u) R_{10}\left(v_{1} q^{-n} / u\right)^{-1} \ldots R_{k 0}\left(v_{k} q^{-n} / u\right)^{-1} .
\end{aligned}
$$

Next, due to (2.7), we have

$$
\begin{aligned}
L_{0}^{-}(u) R_{10}\left(v_{1} q^{-n} / u\right)^{-1} \ldots R_{k 0}\left(v_{k} q^{-n} / u\right)^{-1} L_{1}^{-}\left(v_{1} q^{-n}\right)^{-1} \ldots L_{k}^{-}\left(v_{k} q^{-n}\right)^{-1} \\
\quad=L_{1}^{-}\left(v_{1} q^{-n}\right)^{-1} \ldots L_{k}^{-}\left(v_{k} q^{-n}\right)^{-1} R_{10}\left(v_{1} q^{-n} / u\right)^{-1} \ldots R_{k 0}\left(v_{k} q^{-n} / u\right)^{-1} L_{0}^{-}(u) .
\end{aligned}
$$

The argument is completed by verifying that the trace

$$
\begin{aligned}
\operatorname{tr}_{1, \ldots, k} R_{k 0}\left(v_{k} q^{n} / u\right) \ldots R_{10}\left(v_{1} q^{n} / u\right) & L_{k}^{+}\left(v_{k}\right) \ldots L_{1}^{+}\left(v_{1}\right) L_{1}^{-}\left(v_{1} q^{-n}\right)^{-1} \ldots L_{k}^{-}\left(v_{k} q^{-n}\right)^{-1} \\
& \times R_{10}\left(v_{1} q^{-n} / u\right)^{-1} \ldots R_{k 0}\left(v_{k} q^{-n} / u\right)^{-1} D_{1} \ldots D_{k} A^{(k)}
\end{aligned}
$$

coincides with $\ell_{k}(z)$. This is done in the same way as for the expression (3.10) with the use of Lemma 3.1 and the second crossing symmetry relation in (2.13). 
As a corollary of Theorem 3.2, we obtain an explicit description of invariants of the vacuum module over the quantum affine algebra; cf. [7], [8]. By definition, the vacuum module at the critical level $V_{q}\left(\mathfrak{g l}_{n}\right)$ is the quotient of $\mathrm{U}_{q}\left(\widehat{\mathfrak{g l}}_{n}\right)_{\text {cri }}$ by the left ideal generated by all elements $l_{i j}^{-}[r]$ with $r>0$ and by the elements $l_{i j}^{-}[0]-\delta_{i j}$ with $i \geqslant j$. The module $V_{q}\left(\mathfrak{g l}_{n}\right)$ is generated by the vector $\mathbf{1}$ (the image of $1 \in \mathrm{U}_{q}\left(\widehat{\mathfrak{g l}}_{n}\right)_{\text {cri }}$ in the quotient) such that

$$
L^{-}(u) \mathbf{1}=I \mathbf{1}
$$

where $I$ denotes the identity matrix. As a vector space, $V_{q}\left(\mathfrak{g l}_{n}\right)$ can be identified with the subalgebra $\mathrm{Y}_{q}\left(\mathfrak{g l}_{n}\right)$ of $\mathrm{U}_{q}\left(\widehat{\mathfrak{g l}}_{n}\right)_{\text {cri }}$ generated by the coefficients of all series $l_{i j}^{+}(u)$ subject to the additional relations $l_{i i}^{+}[0]=1$. This relies on the Poincaré-Birkhoff-Witt theorem for the quantum affine algebra; see, e.g., Sec. 5 below. The subspace of invariants of $V_{q}\left(\mathfrak{g l}_{n}\right)$ is defined by

$$
\mathfrak{z}_{q}\left(\widehat{\mathfrak{g l}}_{n}\right)=\left\{v \in V_{q}\left(\mathfrak{g l}_{n}\right) \mid L^{-}(u) v=I v\right\} ;
$$

cf. [15, Sec. 3.3] and [18, Sec. 8]. One can regard $\mathfrak{z}_{q}\left(\widehat{\mathfrak{g l}}_{n}\right)$ as a subspace of $\mathrm{Y}_{q}\left(\mathfrak{g l}_{n}\right)$. Moreover, this subspace is closed under the multiplication in the quantum affine algebra. Therefore, $\mathfrak{z}_{q}\left(\widehat{\mathfrak{g l}}_{n}\right)$ can be identified with a subalgebra of $\mathrm{Y}_{q}\left(\mathfrak{g l}_{n}\right)$. For $k=1, \ldots, n$ introduce the series $\bar{\ell}_{k}(z)$ with coefficients in $\mathrm{Y}_{q}\left(\mathfrak{g l}_{n}\right)$ by

$$
\bar{\ell}_{k}(z)=\operatorname{tr}_{1, \ldots, k} A^{(k)} L_{1}^{+}(z) \ldots L_{k}^{+}\left(z q^{-2 k+2}\right) D_{1} \ldots D_{k}
$$

Corollary 3.3. All coefficients of the series $\bar{\ell}_{k}(z) \mathbf{1}$ with $k=1, \ldots, n$ belong to the algebra of invariants $\mathfrak{z}_{q}\left(\widehat{\mathfrak{g l}}_{n}\right)$. Moreover, the coefficients of all series $\bar{\ell}_{k}(z)$ pairwise commute.

Proof. By Theorem 3.2, we have $L^{-}(u) \ell_{k}(z)=\ell_{k}(z) L^{-}(u)$. Apply both sides to the vector $\mathbf{1} \in V_{q}\left(\mathfrak{g l}_{n}\right)$ and observe that $\ell_{k}(z) \mathbf{1}=\bar{\ell}_{k}(z) \mathbf{1}$. This proves the first part of the corollary. The second part follows by the application of both sides of the identity $\ell_{k}(z) \ell_{m}(w)=$ $\ell_{m}(w) \ell_{k}(z)$ to the vector $\mathbf{1}$. For the left hand side we get

$$
\ell_{k}(z) \ell_{m}(w) \mathbf{1}=\ell_{k}(z) \bar{\ell}_{m}(w) \mathbf{1}=\bar{\ell}_{m}(w) \ell_{k}(z) \mathbf{1}=\bar{\ell}_{m}(w) \bar{\ell}_{k}(z) \mathbf{1}
$$

The same calculation for the right hand side gives $\bar{\ell}_{k}(z) \bar{\ell}_{m}(w)=\bar{\ell}_{m}(w) \bar{\ell}_{k}(z)$.

The second part of the corollary is well known; the series $\bar{\ell}_{k}(z)$ essentially coincides with the transfer matrix associated with the $k$-th fundamental representation of $\mathrm{U}_{q}\left(\widehat{\mathfrak{g l}}_{n}\right)$; see e.g. [18]. The Harish-Chandra image of $\bar{\ell}_{k}(z)$ coincides with the $q$-character of this representation; see also Theorem 6.2 below which recovers the calculation of the image in a more general context.

Remark 3.4. The form of the series $\ell_{k}(z)$ and $\bar{\ell}_{k}(z)$ indicates a possible interpretation of their properties from the viewpoint of the quantum vertex algebra theory of [12]. 


\section{Quantum minor formulas for $\ell_{k}(z)$ and $\bar{\ell}_{k}(z)$}

By calculating the trace in (3.4), we can get two quantum minor-type expressions for $\ell_{k}(z)$ in terms of the entries of the matrices $L^{+}(z)=\left[l_{i j}^{+}(z)\right]$ and $\widetilde{L}(z):=L^{-}(z)^{-1} D=\left[\widetilde{l}_{i j}(z)\right]$. We will denote by $l(\sigma)$ the length of a reduced decomposition of a permutation $\sigma \in \mathfrak{S}_{k}$. The length $l(\sigma)$ coincides with the number of inversions in the sequence $(\sigma(1), \ldots, \sigma(k))$.

Proposition 4.1. For $k=1, \ldots, n$ we have

$$
\begin{aligned}
\ell_{k}(z)=\sum_{j_{1}, \ldots, j_{k}} \sum_{i_{1}<\cdots<i_{k}} \sum_{\sigma \in \mathfrak{S}_{k}}(-q)^{-l(\sigma)} l_{i_{\sigma(1)} j_{1}}^{+}(z) \ldots l_{i_{\sigma(k)} j_{k}}^{+}\left(z q^{-2 k+2}\right) & \\
& \times \widetilde{l}_{j_{k} i_{k}}\left(z q^{-n-2 k+2}\right) \ldots \widetilde{l}_{j_{1} i_{1}}\left(z q^{-n}\right)
\end{aligned}
$$

and

$$
\begin{aligned}
\ell_{k}(z)=\sum_{j_{1}, \ldots, j_{k}} \sum_{i_{1}<\cdots<i_{k}} \sum_{\sigma \in \mathfrak{S}_{k}}(-q)^{l(\sigma)} l_{i_{\sigma(k)} j_{k}}^{+}(z) \ldots l_{i_{\sigma(1)} j_{1}}^{+}\left(z q^{-2 k+2}\right) & \\
& \times \widetilde{l}_{j_{1} i_{1}}\left(z q^{-n-2 k+2}\right) \ldots \widetilde{l}_{j_{k} i_{k}}\left(z q^{-n}\right) .
\end{aligned}
$$

Proof. Using (3.4) we interpret

$$
A^{(k)} L_{1}^{+}\left(v_{1}\right) \ldots L_{k}^{+}\left(v_{k}\right) \widetilde{L}_{k}\left(v_{k} q^{-n}\right) \ldots \widetilde{L}_{1}\left(v_{1} q^{-n}\right)
$$

with $v_{a}=z q^{-2 a+2}$ as an operator in the vector space $\left(\mathbb{C}^{n}\right)^{\otimes k}$. Since it is divisible on the right by $A^{(k)}$, the trace of the operator can be found as $k$ ! times the sum of the diagonal matrix elements corresponding to basis vectors of the form $e_{i_{1}} \otimes \ldots \otimes e_{i_{k}}$ with $i_{1}<\cdots<i_{k}$. Now (4.1) follows with the use of (3.3) and the action of the $q$-permutations on the basis vectors of this form: for any $\sigma \in \mathfrak{S}_{k}$

$$
P_{\sigma}^{q}\left(e_{i_{1}} \otimes \ldots \otimes e_{i_{k}}\right)=q^{l(\sigma)} e_{i_{\sigma^{-1}(1)}} \otimes \ldots \otimes e_{i_{\sigma^{-1}(k)}}
$$

and hence

$$
P_{\sigma}^{q}\left(e_{i_{\sigma(1)}} \otimes \ldots \otimes e_{i_{\sigma(k)}}\right)=q^{-l(\sigma)} e_{i_{1}} \otimes \ldots \otimes e_{i_{k}} .
$$

The proof of (4.2) is quite similar; use the basis vectors $e_{i_{k}} \otimes \ldots \otimes e_{i_{1}}$ with the same condition $i_{1}<\cdots<i_{k}$ on the indices.

Remark 4.2. Two more formulas for $\ell_{k}(z)$ analogous to (4.1) and (4.2) can be obtained by using (3.8) instead of (3.4).

The series $\ell_{n}(z)$ can be factorized into a product of two quantum determinants. To derive the factorization formula, recall a construction of the quantum minors of the matrices $L^{ \pm}(z)$. Lemma 3.1 implies the relations

$$
A^{(k)} L_{1}^{ \pm}(z) \ldots L_{k}^{ \pm}\left(q^{-2 k+2} z\right)=L_{k}^{ \pm}\left(q^{-2 k+2} z\right) \ldots L_{1}^{ \pm}(z) A^{(k)} .
$$


The quantum minors $L^{ \pm}(z)_{b_{1} \cdots b_{k}}^{a_{1} \cdots a_{k}}$ are the coefficients in the expansion of the either side of (4.3) along the basis of matrix units:

$$
\sum_{a_{i}, b_{i}} e_{a_{1} b_{1}} \otimes \ldots \otimes e_{a_{k} b_{k}} \otimes L^{ \pm}(z)_{b_{1} \ldots b_{k}}^{a_{1} \ldots a_{k}}
$$

The following formulas are immediate from the definition. If $a_{1}<\cdots<a_{k}$ then

$$
L^{ \pm}(z)_{b_{1} \cdots b_{k}}^{a_{1} \cdots a_{k}}=\sum_{\sigma \in \mathfrak{S}_{k}}(-q)^{-l(\sigma)} l_{a_{\sigma(1)} b_{1}}^{ \pm}(z) \cdots l_{a_{\sigma(k)} b_{k}}^{ \pm}\left(q^{-2 k+2} z\right)
$$

and for any $\tau \in \mathfrak{S}_{k}$ we have

$$
L^{ \pm}(z)_{b_{1} \ldots b_{k}}^{a_{\tau(1)} \ldots a_{\tau(k)}}=(-q)^{l(\tau)} L^{ \pm}(z)_{b_{1} \ldots b_{k}}^{a_{1} \ldots a_{k}}
$$

If $b_{1}<\cdots<b_{k}$ (and the $a_{i}$ are arbitrary) then

$$
L^{ \pm}(z)_{b_{1} \ldots b_{k}}^{a_{1} \ldots a_{k}}=\sum_{\sigma \in \mathfrak{S}_{k}}(-q)^{l(\sigma)} l_{a_{k} b_{\sigma(k)}}^{ \pm}\left(q^{-2 k+2} z\right) \ldots l_{a_{1} b_{\sigma(1)}}^{ \pm}(z)
$$

and for any $\tau \in \mathfrak{S}_{k}$ we have

$$
L^{ \pm}(z)_{b_{\tau(1) \ldots b_{\tau(k)}}}^{a_{1} \ldots a_{k}}=(-q)^{-l(\tau)} L^{ \pm}(z)_{b_{1} \ldots b_{k}}^{a_{1} \ldots a_{k}}
$$

Moreover, the quantum minor is zero if two top or two bottom indices are equal.

The following lemma is well-known. We give a proof for completeness 1

Lemma 4.3. The coefficients of the quantum determinants

$$
\operatorname{qdet} L^{ \pm}(z)=L^{ \pm}(z)_{1 \cdots n}^{1 \cdots n}
$$

belong to the center of the quantum affine algebra $\mathrm{U}_{q}\left(\widehat{\mathfrak{g l}}_{n}\right)_{\text {cri }}$ at the critical level.

Proof. Introduce the product

$$
R\left(v_{0}, v_{1}, \ldots, v_{n}\right)=\prod_{0 \leqslant a<b \leqslant n} R_{a b}\left(v_{a} / v_{b}\right),
$$

where the $v_{a}$ are variables and the product is taken in the lexicographical order on the pairs $(a, b)$. The defining relations (2.7) and (2.8) imply

$$
R\left(u q^{n}, v_{1}, \ldots, v_{n}\right) L_{0}^{+}(u) L_{1}^{-}\left(v_{1}\right) \ldots L_{n}^{-}\left(v_{n}\right)=L_{n}^{-}\left(v_{n}\right) \ldots L_{1}^{-}\left(v_{1}\right) L_{0}^{+}(u) R\left(u q^{-n}, v_{1}, \ldots, v_{n}\right) .
$$

\footnotetext{
${ }^{1}$ The critical level assumption $k=-2$ is omitted in the corresponding statement in [17, Lemma 2] in the case $n=2$. It was only used there under this assumption.
} 
Use (2.1)-(2.3) to write this relation in terms of the $R$-matrix $R(u, v)$. By cancelling common factors we get

$$
\widetilde{R}\left(u q^{n}, v_{1}, \ldots, v_{n}\right) L_{0}^{+}(u) L_{1}^{-}\left(v_{1}\right) \ldots L_{n}^{-}\left(v_{n}\right)=L_{n}^{-}\left(v_{n}\right) \ldots L_{1}^{-}\left(v_{1}\right) L_{0}^{+}(u) \widetilde{R}\left(u q^{-n}, v_{1}, \ldots, v_{n}\right),
$$

where

$$
\widetilde{R}\left(v_{0}, v_{1}, \ldots, v_{n}\right)=\prod_{a=1}^{n} \frac{f\left(v_{0} / v_{a}\right)}{q^{-1} v_{0}-q v_{a}} \prod_{0 \leqslant a<b \leqslant n} R_{a b}\left(v_{a}, v_{b}\right) .
$$

Now specialize the variables by setting $v_{a}=z q^{-2 a+2}$ for $a=1, \ldots, n$ and replace the product of $R$-matrices $R_{a b}\left(v_{a}, v_{b}\right)$ over the set of pairs $1 \leqslant a<b \leqslant n$ using Lemma 3.1. Since

$$
A^{(n)} L_{1}^{ \pm}\left(v_{1}\right) \ldots L_{n}^{ \pm}\left(v_{n}\right)=L_{n}^{ \pm}\left(v_{n}\right) \ldots L_{1}^{ \pm}\left(v_{1}\right) A^{(n)}=A^{(n)} \operatorname{qdet} L^{ \pm}(z)
$$

we get

$$
\begin{aligned}
\prod_{a=1}^{n} \frac{f\left(u q^{n} / v_{a}\right)}{u q^{n-1}-q v_{a}} \prod_{a=1, \ldots, n} & R_{0 a}\left(u q^{n}, v_{a}\right) A^{(n)} L_{0}^{+}(u) \operatorname{qdet} L^{-}(z) \\
& =\prod_{a=1}^{n} \frac{f\left(u q^{-n} / v_{a}\right)}{u q^{-n-1}-q v_{a}} \operatorname{qdet} L^{-}(z) L_{0}^{+}(u) A^{(n)} \prod_{a=1, \ldots, n} R_{0 a}\left(u q^{-n}, v_{a}\right) .
\end{aligned}
$$

Observe that

$$
\prod_{a=1, \ldots, n} R_{0 a}\left(v_{0}, v_{a}\right) A^{(n)}=A^{(n)} \prod_{a=1, \ldots, n} R_{0 a}\left(v_{0}, v_{a}\right)=A^{(n)}\left(q^{-1} v_{0}-q v_{1}\right) \prod_{a=2}^{n}\left(v_{0}-v_{a}\right)
$$

Indeed, by the first equality, it suffices to verify the second equality on the basis vectors of the form $e_{i} \otimes e_{i} \otimes e_{1} \otimes \ldots \otimes e_{i-1} \otimes e_{i-1} \otimes \ldots \otimes e_{n}$ for $i=1, \ldots, n$ which is straightforward. Thus, we can conclude that $L_{0}^{+}(u)$ commutes with qdet $L^{-}(z)$ due to the identity

$$
\prod_{a=1}^{n} \frac{f\left(u q^{n} / v_{a}\right)}{f\left(u q^{-n} / v_{a}\right)}=\prod_{a=2}^{n} \frac{\left(u q^{n-1}-q v_{a}\right)\left(u q^{-n}-v_{a}\right)}{\left(u q^{-n-1}-q v_{a}\right)\left(u q^{n}-v_{a}\right)}
$$

which follows from (2.4). The relation $L_{0}^{-}(u)$ qdet $L^{+}(z)=\operatorname{qdet} L^{+}(z) L_{0}^{-}(u)$ is verified by a similar argument with the use of the unitarity property of the $R$-matrix (2.2):

$$
\bar{R}\left(x^{-1}\right)=\bar{R}_{21}(x)^{-1} .
$$

The proof of the remaining two relations $L_{0}^{ \pm}(u) \operatorname{qdet} L^{ \pm}(z)=\operatorname{qdet} L^{ \pm}(z) L_{0}^{ \pm}(u)$ is simpler as it relies only on the defining relations (2.7).

Remark 4.4. Both quantum determinants (4.9) are also known to be central in the quantum affine algebra $\mathrm{U}_{q}\left(\widehat{\mathfrak{g l}}_{n}\right)$ at the zero level $c=0$; the algebra is defined as in Sec. 2 with the factor $f(x)$ in (2.2) omitted. 
Corollary 4.5. We have

$$
\ell_{n}(z)=\operatorname{qdet} L^{+}(z)\left(\operatorname{qdet} L^{-}\left(z q^{-n}\right)\right)^{-1}
$$

Proof. Relation (4.10) implies

$$
A^{(n)} L_{n}^{-}\left(v_{n}\right)^{-1} \ldots L_{1}^{-}\left(v_{1}\right)^{-1}=\left(\operatorname{qdet} L^{-}(z)\right)^{-1} A^{(n)} .
$$

Replacing here $z$ by $z q^{-n}$ and using qdet $D=1$ we get the desired formula from (3.4).

We will now give a formulation of Corollary 3.3 by combining the series $\bar{\ell}_{k}(z)$ defined in (3.15) into a single determinant by some analogy with [7] and [8]. Introduce the extension $\mathrm{Y}_{q}^{\text {ext }}\left(\mathfrak{g l}_{n}\right)$ of the algebra $\mathrm{Y}_{q}\left(\mathfrak{g l}_{n}\right)$ by adjoining pairwise commuting elements $\pi_{1}, \ldots, \pi_{n}$ subject to the additional relations

$$
\pi_{i} l_{k m}^{+}(u)= \begin{cases}l_{k m}^{+}(u) \pi_{i} & \text { if } i \leqslant k, \\ q^{2} l_{k m}^{+}(u) \pi_{i} & \text { if } i>k\end{cases}
$$

Combine the elements $\pi_{i}$ into the diagonal matrix $\Pi=\operatorname{diag}\left[\pi_{1}, \ldots, \pi_{n}\right]$. We have a vector space isomorphism $\mathrm{Y}_{q}^{\text {ext }}\left(\mathfrak{g l}_{n}\right) / J \cong \mathrm{Y}_{q}\left(\mathfrak{g l}_{n}\right)$, where $J$ is the left ideal of $\mathrm{Y}_{q}^{\text {ext }}\left(\mathfrak{g l}_{n}\right)$ generated by the elements $\pi_{i}-1$ with $i=1, \ldots, n$. We will identify $\mathrm{Y}_{q}\left(\mathfrak{g l}_{n}\right)$ with the quotient via this isomorphism.

We will point out a connection with $q$-analogues of Manin matrices (also known as right quantum matrices); see, e.g., [6] for a detailed account of their properties. An $n \times n$ matrix $M$ with entries in an associative algebra $\mathcal{A}$ is called $q$-Manin if it satisfies the relation

$$
A^{(2)} M_{1} M_{2}=A^{(2)} M_{1} M_{2} A^{(2)}
$$

in the algebra End $\mathbb{C}^{n} \otimes$ End $\mathbb{C}^{n} \otimes \mathcal{A}$ with the meaning of the subscripts as in (2.11).

Introduce the operator $\delta$ which interacts with power series in $z$ by the rule $\delta g(z)=$ $g\left(z q^{-2}\right) \delta$. Adjoining this element to the algebra $\mathrm{Y}_{q}\left(\mathfrak{g l}_{n}\right)$ we find that both $L^{+}(z) \delta$ and $L^{+}(z) D \delta$ are $q$-Manin matrices. Define the $q$-determinant of a square matrix $M$ by

$$
\operatorname{det}_{q} M=\sum_{\sigma \in \mathfrak{S}_{n}}(-q)^{-l(\sigma)} M_{\sigma(1) 1} \cdots M_{\sigma(n) n} .
$$

In the following proposition we regard the $q$-determinant of the matrix $\Pi+L^{+}(z) D \delta$ as a polynomial in $\delta$ with coefficients in $\mathrm{Y}_{q}^{\mathrm{ext}}\left(\mathfrak{g l}_{n}\right)$.

Proposition 4.6. We have the relation modulo the left ideal J:

$$
\operatorname{det}_{q}\left(\Pi+L^{+}(z) D \delta\right)=1+\sum_{k=1}^{n} \bar{\ell}_{k}(z) \delta^{k}
$$


Proof. Calculating the $q$-determinant we will write it as the sum of monomials of the form

$$
\begin{aligned}
(-q)^{-l(\sigma)} M_{\sigma(1) 1} \cdots & M_{\sigma\left(i_{1}-1\right) i_{1}-1} \pi_{i_{1}} M_{\sigma\left(i_{1}+1\right) i_{1}+1} \\
& \times \cdots M_{\sigma\left(i_{2}-1\right) i_{2}-1} \pi_{i_{2}} M_{\sigma\left(i_{2}+1\right) i_{2}+1} \cdots \pi_{i_{n-k}} M_{\sigma\left(i_{n-k}+1\right) i_{n-k}+1} \cdots M_{\sigma(n) n}
\end{aligned}
$$

where $M=L^{+}(z) D \delta$ and $\sigma\left(i_{a}\right)=i_{a}$ for $a=1, \ldots, n-k$. Now use relations (4.11) to move all the elements $\pi_{i_{a}}$ so they will appear to the right from all factors $M_{k m}$ in the monomial. As a result, by moving each element $\pi_{i_{a}}$ we get the factor $q^{2 r_{a}}$, where $r_{a}$ is the number of elements of the set $\left\{\sigma\left(i_{a}+1\right), \ldots, \sigma(n)\right\}$ which are less than $i_{a}$. That is, $r_{a}$ is the number of inversions formed by the index $i_{a}$ with the indices of the set $\left\{\sigma\left(i_{a}+1\right), \ldots, \sigma(n)\right\}$. Therefore, after moving all the elements $\pi_{i_{a}}$, the monomial will get the factor $(-q)^{-l(\widehat{\sigma})}$, where $\widehat{\sigma}$ is the sequence of elements obtained from $(\sigma(1), \ldots, \sigma(n))$ by removing $i_{1}, \ldots, i_{n-k}$, and $l(\widehat{\sigma})$ is the number of inversions in that sequence. This demonstrates that the coefficient of the product $\pi_{i_{1}} \ldots \pi_{i_{n-k}}$ coincides with the $q$-determinant of the principal submatrix of $M$ obtained by deleting rows and columns enumerated by $i_{1}, \ldots, i_{n-k}$. This $q$-determinant equals $\left[L^{+}(z) D\right]_{a_{1} \cdots a_{k}}^{a_{1} \cdots a_{k}} \delta^{k}$ where $\left\{a_{1}, \ldots, a_{k}\right\}=\{1, \ldots, n\} \backslash\left\{i_{1}, \ldots, i_{n-k}\right\}$. Since

$$
\bar{\ell}_{k}(z)=\sum_{1 \leqslant a_{1}<\cdots<a_{k} \leqslant n}\left[L^{+}(z) D\right]_{a_{1} \cdots a_{k}}^{a_{1} \cdots a_{k}}
$$

the required relation follows by taking the quotient over the left ideal $J$.

The next lemma will be used in Sec. 6 below.

Lemma 4.7. The entries of the inverse matrix $L^{-}(z)^{-1}$ are found by the formula

$$
\left[L^{-}(z)^{-1}\right]_{i j}=(-q)^{j-i}\left(q \operatorname{qdet} L^{-}\left(z q^{2 n-2}\right)\right)^{-1} L^{-}\left(z q^{2 n-2}\right)_{1 \ldots . \ldots n}^{1 \ldots \ldots},
$$

where the hats indicate indices to be skipped.

Proof. By (4.10) we have

$$
A^{(n)} L_{1}^{-}\left(v_{1}\right) \ldots L_{n-1}^{-}\left(v_{n-1}\right)=A^{(n)} \operatorname{qdet} L^{-}(z) L_{n}^{-}\left(v_{n}\right)^{-1},
$$

where $v_{a}=z q^{-2 a+2}$, as before. The desired formula follows by the application of both sides to the basis vector $e_{1} \otimes \ldots \otimes \widehat{e_{i}} \otimes \ldots \otimes e_{n} \otimes e_{j}$ and the replacement $z \mapsto z q^{2 n-2}$.

\section{Poincaré-Birkhoff-Witt theorem}

To define analogues of the Harish-Chandra homomorphism, we will need a version of the Poincaré-Birkhoff-Witt theorem for the quantum affine algebra $\mathrm{U}_{q}\left(\widehat{\mathfrak{g l}}_{n}\right)$. Introduce a total ordering $\prec$ on the set of generators as follows. First, each generator $l_{i j}^{+}[r]$ precedes each 
generator $l_{k m}^{-}[s]$. Furthermore, $l_{i j}^{+}[r] \prec l_{k m}^{+}[s]$ if and only if the triple $(j-i, i, r)$ precedes $(m-k, k, s)$ in the lexicographical order. Finally, we set $l_{i j}^{-}[r] \prec l_{k m}^{-}[s]$ if and only if the triple $(i-j, i, r)$ precedes $(k-m, k, s)$ in the lexicographical order. Note that by the defining relations (2.7),

$$
\left[l_{i j}^{ \pm}[r], l_{i j}^{ \pm}[s]\right]=0
$$

for all $r$ and $s$. Hence, the ordering $\prec$ induces a well-defined total ordering on the series (2.9) such that $l_{i j}^{+}(u) \prec l_{k m}^{-}(u)$ and

$$
\begin{aligned}
l_{n 1}^{+}(u) \prec l_{n-11}^{+}(u) \prec l_{n 2}^{+}(u) \prec \cdots \prec l_{11}^{+}(u) \prec \cdots \prec l_{n n}^{+}(u) \prec l_{12}^{+}(u) \prec \cdots \prec l_{1 n}^{+}(u), \\
l_{1 n}^{-}(u) \prec l_{1 n-1}^{-}(u) \prec l_{2 n}^{-}(u) \prec \cdots \prec l_{11}^{-}(u) \prec \cdots \prec l_{n n}^{-}(u) \prec l_{21}^{-}(u) \prec \cdots \prec l_{n 1}^{-}(u) .
\end{aligned}
$$

Consider the ordered monomials in the generators $l_{i j}^{ \pm}[r]$ multiplied by integer powers of the central element $\gamma=q^{c}$ (the zero elements $l_{i j}^{+}[0]$ for $i>j$ and $l_{i j}^{-}[0]$ for $i<j$ are excluded). Relations (2.7) and (2.8) imply that

$$
l_{i i}^{+}[0] l_{k m}^{ \pm}(u)=q^{-\delta_{i k}+\delta_{i m}} l_{k m}^{ \pm}(u) l_{i i}^{+}[0] \quad \text { and } \quad l_{i i}^{-}[0] l_{k m}^{ \pm}(u)=q^{\delta_{i k}-\delta_{i m}} l_{k m}^{ \pm}(u) l_{i i}^{-}[0] .
$$

Hence, using (2.6) we may suppose that for each $i=1, \ldots, n$ each monomial only contains either a nonnegative power of $l_{i i}^{+}[0]$ or a positive power of $l_{i i}^{-}[0]$. Under these assumptions we have the following version of the Poincaré-Birkhoff-Witt theorem.

Proposition 5.1. The ordered monomials in the generators form a basis of the quantum affine algebra $\mathrm{U}_{q}\left(\widehat{\mathfrak{g l}}_{n}\right)$.

Proof. First, we prove the claim for the quantum affine algebra $\overline{\mathrm{U}}_{q}\left(\widehat{\mathfrak{g l}}_{n}\right)$ which is defined in the same way as $\mathrm{U}_{q}\left(\widehat{\mathfrak{g l}}_{n}\right)$; the only difference is the use of the $R$-matrix (2.2) instead of (2.3). Thus, we only replace (2.8) with the relation

$$
\bar{R}\left(u \gamma^{-1} / v\right) \bar{L}_{1}^{+}(u) \bar{L}_{2}^{-}(v)=\bar{L}_{2}^{-}(v) \bar{L}_{1}^{+}(u) \bar{R}(u \gamma / v)
$$

and leave all other defining relations unchanged. Here we use the bar symbol over the respective objects associated with the algebra $\overline{\mathrm{U}}_{q}\left(\widehat{\mathfrak{g l}}_{n}\right)$. We begin by showing that the ordered monomials in the generators of $\overline{\mathrm{U}}_{q}\left(\widehat{\mathfrak{g l}}_{n}\right)$ span the algebra. Given a monomial in the generators we will use the induction on its length to show that it equals a linear combination of ordered monomials. Writing (5.3) in terms of the entries of the matrices $\bar{L}^{ \pm}(u)$ we get

$$
\begin{gathered}
\left(\gamma q^{-\delta_{j m}} u-q^{\delta_{j m}} v\right) \bar{l}_{k m}^{-}(v) \bar{l}_{i j}^{+}(u)+\left(q^{-1}-q\right)\left(u \gamma \delta_{m>j}+v \delta_{m<j}\right) \bar{l}_{k j}^{-}(v) \bar{l}_{i m}^{+}(u) \\
=\left[\left(\gamma^{-1} q^{-\delta_{i k}} u-q^{\delta_{i k}} v\right) \bar{l}_{i j}^{+}(u) \bar{l}_{k m}^{-}(v)\right. \\
\left.\quad+\left(q^{-1}-q\right)\left(u \gamma^{-1} \delta_{i>k}+v \delta_{i<k}\right) \bar{l}_{k j}^{+}(u) \bar{l}_{i m}^{-}(v)\right] \frac{\gamma q^{-2} u-v}{\gamma^{-1} q^{-2} u-v}
\end{gathered}
$$


where $\delta_{i<j}$ or $\delta_{i>j}$ equals 1 if the subscript inequality is satisfied and 0 otherwise. If $m=j$ then the relation allows us to write $\bar{l}_{k j}^{-}[s] \bar{l}_{i j}^{+}[r]$ as a linear combination of ordered products of generators. If $m \neq j$ then we swap $m$ and $j$ in the relation to get a system of two equations for $\bar{l}_{k m}^{-}(v) \bar{l}_{i j}^{+}(u)$ and $\bar{l}_{k j}^{-}(v) \bar{l}_{i m}^{+}(u)$. By solving the system we will be able to write $\bar{l}_{k m}^{-}[s] \bar{l}_{i j}^{+}[r]$ as a linear combination of ordered products of generators.

Similarly, (2.7) gives the relation

$$
\begin{aligned}
& \left(q^{-\delta_{i k}} u-q^{\delta_{i k}} v\right) \bar{l}_{i j}^{ \pm}(u) \bar{l}_{k m}^{ \pm}(v)+\left(q^{-1}-q\right)\left(u \delta_{i>k}+v \delta_{i<k}\right) \bar{l}_{k j}^{ \pm}(u) \bar{l}_{i m}^{ \pm}(v) \\
& =\left(q^{-\delta_{j m}} u-q^{\delta_{j m}} v\right) \bar{l}_{k m}^{ \pm}(v) \bar{l}_{i j}^{ \pm}(u)+\left(q^{-1}-q\right)\left(u \delta_{m>j}+v \delta_{m<j}\right) \bar{l}_{k j}^{ \pm}(v) \bar{l}_{i m}^{ \pm}(u)
\end{aligned}
$$

which implies that $\bar{l}_{i j}^{ \pm}[r] \bar{l}_{k m}^{ \pm}[s]$ is a linear combination of ordered products of generators; see [21, Corollary 2.13] for a detailed argument.2]

As a next step, we will show that the ordered monomials are linearly independent in $\overline{\mathrm{U}}_{q}\left(\widehat{\mathfrak{g l}}_{n}\right)$. Consider the quantum affine algebra $\mathrm{U}_{q}\left(\widehat{\mathfrak{g l}}_{n}\right)_{0}$ at the level zero which is the quotient of $\overline{\mathrm{U}}_{q}\left(\widehat{\mathfrak{g l}}_{n}\right)$ by the relation $\gamma=1$. We have the natural epimorphism

$$
\psi: \overline{\mathrm{U}}_{q}\left(\widehat{\mathfrak{g l}}_{n}\right) \rightarrow \mathrm{U}_{q}\left(\widehat{\mathfrak{g l}}_{n}\right)_{0}, \quad \bar{L}^{ \pm}(u) \mapsto L^{ \pm}(u), \quad \gamma \mapsto 1 .
$$

Suppose that a Laurent polynomial in $\gamma$, whose coefficients are nontrivial linear combinations of ordered monomials in the generators $\bar{l}_{i j}^{ \pm}[r]$, is zero in $\overline{\mathrm{U}}_{q}\left(\widehat{\mathfrak{g l}}_{n}\right)$. Multiplying by a power of $\gamma$ if necessary, we get a polynomial in $\gamma$ equal to zero. Choose such a polynomial $P=x_{k} \gamma^{k}+\cdots+x_{0}$ of the minimal possible degree $k \geqslant 0$. Since $P=0$ in $\overline{\mathrm{U}}_{q}\left(\widehat{\mathfrak{g l}}_{n}\right)$ we have

$$
0=\psi(P)=\psi\left(x_{k}\right)+\cdots+\psi\left(x_{0}\right)=\psi\left(x_{k}+\cdots+x_{0}\right) .
$$

The sum $x=x_{k}+\cdots+x_{0}$ is a linear combination of ordered monomials in the generators $\bar{l}_{i j}^{ \pm}[r]$. By the definition of $\psi$, the image $\psi(x)$ is the corresponding linear combination of ordered monomials in the generators $l_{i j}^{ \pm}[r]$ of $\mathrm{U}_{q}\left(\widehat{\mathfrak{g l}}_{n}\right)_{0}$. On the other hand, by the arguments of [21, Sec. 2.3] applied to this particular ordering, the corresponding version of the Poincaré-Birkhoff-Witt theorem holds for $\mathrm{U}_{q}\left(\widehat{\mathfrak{g l}}_{n}\right)_{0}$, so that the ordered monomials are linearly independent. Hence, $\psi(x)=0$ implies $x=0$. If $k=0$ then this is a contradiction. If $k \geqslant 1$ we can write

$$
P=x_{k} \gamma^{k}+\cdots+x_{0}=(\gamma-1)\left(y_{k-1} \gamma^{k-1}+\cdots+y_{0}\right),
$$

where the $y_{i}$ are again linear combinations of ordered monomials in the generators $\bar{l}_{i j}^{ \pm}[r]$. By the results of [10], the algebra $\overline{\mathrm{U}}_{q}\left(\widehat{\mathfrak{g l}}_{n}\right)$ can be defined by the Drinfeld generators. Due to the well-known relationship between the quantum affine algebras associated with $\mathfrak{s l}_{n}$ and $\mathfrak{g l}_{n}$ (see, e.g., [16, Sec. 2.6]), the Poincaré-Birkhoff-Witt theorem for the algebra

\footnotetext{
${ }^{2}$ The generator matrices $T(u)$ and $\bar{T}(u)$ of [21] correspond to $\bar{L}^{-}(u)$ and $\bar{L}^{+}(u)$, respectively.
} 
$\mathrm{U}_{q}\left(\widehat{\mathfrak{s l}}_{n}\right)$ in its Drinfeld presentation [2, 3] implies that the relation $P=0$ is possible only if $y_{k-1} \gamma^{k-1}+\cdots+y_{0}=0$. This contradicts the minimality of the degree $k$ thus completing the proof for $\overline{\mathrm{U}}_{q}\left(\widehat{\mathfrak{g l}}_{n}\right)$.

Finally, we extend the argument to the quantum affine algebra $\mathrm{U}_{q}\left(\widehat{\mathfrak{g l}}_{n}\right)$ defined with the $R$-matrix (2.3) so that (2.8) with $\gamma=q^{c}$ should be used instead of (5.3). This affects only relation (5.4) (for the generators $l_{i j}^{ \pm}[r]$ instead of $\bar{l}_{i j}^{ \pm}[r]$ ) which will now get an extra factor $f\left(u \gamma^{-1} / v\right) / f(u \gamma / v)$ on the right hand side. However, this does not bring any change into the first part of the argument showing that the ordered monomials in the generators span the algebra $\mathrm{U}_{q}\left(\widehat{\mathfrak{g l}}_{n}\right)$.

To prove the linear independence of the ordered monomials, we follow [10, Sec. V] and introduce a homomorphism

$$
\phi: \mathrm{U}_{q}\left(\widehat{\mathfrak{g l}}_{n}\right) \rightarrow \mathcal{H}_{q}(n) \otimes_{\mathbb{C}\left[\gamma, \gamma^{-1}\right]} \overline{\mathrm{U}}_{q}\left(\widehat{\mathfrak{g l}}_{n}\right),
$$

where $\mathcal{H}_{q}(n)$ is the Heisenberg algebra with generators $\gamma$ and $h[r], r \in \mathbb{Z}, r \neq 0$. The defining relations of $\mathcal{H}_{q}(n)$ have the form

$$
[h[r], h[s]]=\delta_{r,-s} \alpha[r], \quad r \geqslant 1,
$$

and $\gamma$ is central and invertible; all other pairs of the generators commute. The elements $\alpha[r]$ are defined by the expansion

$$
\exp \sum_{r=1}^{\infty} \alpha[r] x^{r}=\frac{f(x \gamma)}{f\left(x \gamma^{-1}\right)}
$$

So we have the identity

$$
\begin{aligned}
f\left(u \gamma^{-1} / v\right) \exp \left(\sum_{r=1}^{\infty} h[r] u^{r}\right) \exp \left(\sum_{s=1}^{\infty} h[-s] v^{-s}\right) & \\
& =f(u \gamma / v) \exp \left(\sum_{s=1}^{\infty} h[-s] v^{-s}\right) \exp \left(\sum_{r=1}^{\infty} h[r] u^{r}\right) .
\end{aligned}
$$

Clearly, the monomials of the form $h\left[r_{1}\right] \ldots h\left[r_{k}\right]$ with $k \geqslant 0$ and $r_{1} \geqslant \cdots \geqslant r_{k}\left(\right.$ with $\left.r_{i} \neq 0\right)$ form a basis of the $\mathbb{C}\left[\gamma, \gamma^{-1}\right]$-module $\mathcal{H}_{q}(n)$. The homomorphism (5.6) is now defined by $\phi: \gamma \mapsto \gamma$ and

$$
\phi: L^{+}(u) \mapsto \exp \left(\sum_{r=1}^{\infty} h[r] u^{r}\right) \bar{L}^{+}(u), \quad L^{-}(u) \mapsto \exp \left(\sum_{r=1}^{\infty} h[-r] u^{-r}\right) \bar{L}^{-}(u) .
$$

Suppose there is a linear combination of the ordered monomials in the generators of $\mathrm{U}_{q}\left(\widehat{\mathfrak{g l}}_{n}\right)$ equal to zero. Consider its image under the homomorphism $\phi$. Using the basis $\left\{h\left[r_{1}\right] \ldots h\left[r_{k}\right]\right\}$ of $\mathcal{H}_{q}(n)$ and the Poincaré-Birkhoff-Witt basis for the algebra $\overline{\mathrm{U}}_{q}\left(\widehat{\mathfrak{g l}}_{n}\right)$, we conclude that all coefficients of the linear combination must be zero. 
We will also need a version of the Poincaré-Birkhoff-Witt theorem for a different ordering of the generators. As we pointed out above, a total ordering can be defined on the generating series (2.9) due to (5.1). We set $l_{i j}^{+}(u) \prec l_{k m}^{-}(u)$ as before, but the remaining conditions are swapped between $l_{i j}^{+}(u)$ and $l_{i j}^{-}(u)$ :

$$
\begin{aligned}
l_{1 n}^{+}(u) \prec l_{1 n-1}^{+}(u) \prec l_{2 n}^{+}(u) \prec \cdots \prec l_{11}^{+}(u) \prec \cdots \prec l_{n n}^{+}(u) \prec l_{21}^{+}(u) \prec \cdots \prec l_{n 1}^{+}(u), \\
l_{n 1}^{-}(u) \prec l_{n-11}^{-}(u) \prec l_{n 2}^{-}(u) \prec \cdots \prec l_{11}^{-}(u) \prec \cdots \prec l_{n n}^{-}(u) \prec l_{12}^{-}(u) \prec \cdots \prec l_{1 n}^{-}(u) .
\end{aligned}
$$

Under the same assumptions on the monomials as for Proposition 5.1, the following holds.

Proposition 5.2. The ordered monomials in the generators form a basis of the quantum affine algebra $\mathrm{U}_{q}\left(\widehat{\mathfrak{g l}}_{n}\right)$.

Proof. The argument is the same as for Proposition 5.1 with some obvious minor changes taking into the account the ordering conditions.

\section{Harish-Chandra homomorphisms}

Consider the quantum affine algebra $\mathrm{U}_{q}\left(\widehat{\mathfrak{g l}}_{n}\right)_{\text {cri }}$ at the critical level, $\gamma=q^{-n}$. By Proposition [5.1, any element $x \in \mathrm{U}_{q}\left(\widehat{\mathfrak{g l}}_{n}\right)_{\text {cri }}$ can be written as a unique linear combination of ordered monomials in the generators $l_{i j}^{ \pm}[r]$. Denote by $\mathrm{U}^{0}$ the subspace of the algebra spanned by those monomials which do not contain any generators $l_{i j}^{ \pm}[r]$ with $i \neq j$. Let $x_{0}$ denote the component of the linear combination representing the element $x$, which belongs to $\mathrm{U}^{0}$. The mapping $\theta: x \mapsto x_{0}$ defines the projection $\theta: \mathrm{U}_{q}\left(\widehat{\mathfrak{g l}}_{n}\right)_{\text {cri }} \rightarrow \mathrm{U}^{0}$. Extending it by continuity we get the projection $\theta: \widetilde{\mathrm{U}}_{q}\left(\widehat{\mathfrak{g l}}_{n}\right)_{\text {cri }} \rightarrow \widetilde{\mathrm{U}}^{0}$ to the corresponding completed vector space $\widetilde{U}^{0}$.

Introduce the algebra $\Pi_{q}(n)$ as the quotient of the algebra of polynomials in independent variables $l_{i}^{+}[-r], l_{i}^{-}[r]$ with $i=1, \ldots, n$ and $r=0,1, \ldots$ by the relations $l_{i}^{+}[0] l_{i}^{-}[0]=1$ for

all $i$. The mapping $\eta: \mathrm{U}^{0} \rightarrow \Pi_{q}(n)$ which takes each ordered monomial in the generators $l_{i i}^{ \pm}[\mp r]$ to the corresponding monomial in the variables $l_{i}^{ \pm}[\mp r]$ by the rule $l_{i i}^{ \pm}[\mp r] \mapsto l_{i}^{ \pm}[\mp r]$ extends to an isomorphism of vector spaces. Define the completion $\widetilde{\Pi}_{q}(n)$ of the algebra $\Pi_{q}(n)$ as the inverse limit

$$
\widetilde{\Pi}_{q}(n)=\lim _{\longleftarrow} \Pi_{q}(n) / I_{p}, \quad p>0,
$$

where $I_{p}$ denotes the ideal of $\Pi_{q}(n)$ generated by all elements $l_{i}^{-}[r]$ with $r \geqslant p$; cf. (3.1). The isomorphism $\eta$ extends to an isomorphism of the respective completed vector spaces $\eta: \widetilde{\mathrm{U}}^{0} \rightarrow \widetilde{\Pi}_{q}(n)$. Thus we get a linear map

$$
\chi: \widetilde{\mathrm{U}}_{q}\left(\widehat{\mathfrak{g l}}_{n}\right)_{\mathrm{cri}} \rightarrow \widetilde{\Pi}_{q}(n)
$$

defined as the composition $\chi=\eta \circ \theta$. The next proposition provides an analogue of the Harish-Chandra homomorphism for the quantum affine algebra. 
Proposition 6.1. The restriction of the map (6.1) to the center $\mathrm{Z}_{q}\left(\widehat{\mathfrak{g l}}_{n}\right)$ of the algebra $\widetilde{\mathrm{U}}_{q}\left(\widehat{\mathfrak{g l}}_{n}\right)_{\text {cri }}$ is a homomorphism of commutative algebras

$$
\chi: \mathrm{Z}_{q}\left(\widehat{\mathfrak{g l}}_{n}\right) \rightarrow \widetilde{\Pi}_{q}(n)
$$

Proof. For $x, y \in \mathrm{Z}_{q}\left(\widehat{\mathfrak{g l}}_{n}\right)$ set $x_{0}=\chi(x)$ and $y_{0}=\chi(y)$. Write $y$ as a (possibly infinite) linear combination of ordered monomials in the generators $l_{i j}^{ \pm}[r]$. Suppose that

$$
m=\prod_{a} l_{i_{a} j_{a}}^{+}\left[r_{a}\right] \prod_{b} l_{i_{b} j_{b}}^{-}\left[r_{b}\right]
$$

is an ordered monomial which occurs in the linear combination. Note its property

$$
\sum_{a}\left(i_{a}-j_{a}\right)+\sum_{b}\left(i_{b}-j_{b}\right)=0
$$

implied by (5.2). Suppose that $m \in$ ker $\chi$. Since $x$ is in the center, we have

$$
x m=\prod_{a} l_{i_{a} j_{a}}^{+}\left[r_{a}\right] x \prod_{b} l_{i_{b} j_{b}}^{-}\left[r_{b}\right]
$$

To write $x m$ as a linear combination of ordered monomials we will only need to use the defining relations (2.7) which are also given in (5.5) where the series $\bar{l}_{i j}^{ \pm}(u)$ should be replaced with $l_{i j}^{ \pm}(u)$, respectively. Since the relations (5.5) are homogeneous with respect to the weight parameter $i-j+k-m$, we derive that $x m \in \operatorname{ker} \chi$. Hence a nonzero contribution to the image $\chi(x y)$ can only come from $\chi\left(x y_{0}\right)$, that is, from expressions of the form

$$
\prod_{a} l_{i_{a} i_{a}}^{+}\left[r_{a}\right] x \prod_{b} l_{i_{b} i_{b}}^{-}\left[r_{b}\right]
$$

If $p$ is an ordered monomial which occurs in the linear combination representing $x$ and $\chi(p)=0$, then applying property (6.3) to the monomial $p$ we conclude that

$$
\chi: \prod_{a} l_{i_{a} i_{a}}^{+}\left[r_{a}\right] p \prod_{b} l_{i_{b} i_{b}}^{-}\left[r_{b}\right] \mapsto 0 .
$$

Finally, observe that by the defining relations (5.5), any two generators $l_{i i}^{-}[r]$ and $l_{j j}^{-}[s]$ (resp., $l_{i i}^{+}[r]$ and $l_{j j}^{+}[s]$ ) can be permuted modulo ker $\chi$ within any monomial of the form

$$
\prod_{a} l_{i_{a} i_{a}}^{+}\left[r_{a}\right] \prod_{b} l_{i_{b} i_{b}}^{-}\left[r_{b}\right]
$$

This proves that $\chi(x y)=x_{0} y_{0}$. 
Now we are in a position to calculate the Harish-Chandra images of the higher Sugawara operators provided by Theorem 3.2 . Combine the generators of the algebra $\Pi_{q}(n)$ into the series

$$
l_{i}^{+}(z)=\sum_{r=0}^{\infty} l_{i}^{+}[-r] z^{r}, \quad l_{i}^{-}(z)=\sum_{r=0}^{\infty} l_{i}^{-}[r] z^{-r}
$$

and for $i=1, \ldots, n$ set

$$
\lambda_{i}(z)=q^{n-2 i+1} \frac{l_{i}^{+}(z) l_{1}^{-}\left(z q^{-n+2}\right) \ldots l_{i-1}^{-}\left(z q^{-n+2 i-2}\right)}{l_{1}^{-}\left(z q^{-n}\right) \ldots l_{i}^{-}\left(z q^{-n+2 i-2}\right)} .
$$

This is a Laurent series in $z$ whose coefficients are elements of the completed algebra $\widetilde{\Pi}_{q}(n)$.

Theorem 6.2. For each $k=1, \ldots, n$ the image of the series $\ell_{k}(z)$ under the HarishChandra homomorphism (6.2) is found by

$$
\chi: \ell_{k}(z) \mapsto \sum_{1 \leqslant i_{1}<\cdots<i_{k} \leqslant n} \lambda_{i_{1}}(z) \lambda_{i_{2}}\left(z q^{-2}\right) \ldots \lambda_{i_{k}}\left(z q^{-2 k+2}\right) .
$$

Proof. We will use formula (4.1) for $\ell_{k}(z)$. Apply Lemma 4.7 to express the series $\widetilde{l}_{j i}(z)=$ $q^{n-2 i+1}\left[L^{-}(z)^{-1}\right]_{j i}$ in terms of quantum minors. By (4.8) we have

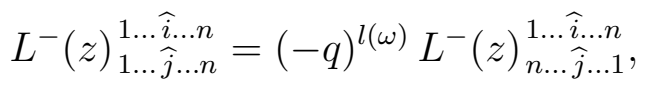

where $\omega \in \mathfrak{S}_{n-1}$ reverses the order of the lower indices. Expanding this quantum minor by (4.5) we find that a nonzero contribution to the image $\chi\left(\ell_{k}(z)\right)$ can only come from the summands in (4.1) with $i_{\sigma(1)} \leqslant j_{1} \leqslant i_{1}$. These conditions imply that $\sigma(1)=1$ and $j_{1}=i_{1}$. By the defining relations in $\mathrm{U}_{q}\left(\widehat{\mathfrak{g l}}_{n}\right)$, the same observation gives $\sigma(2)=2$ and $j_{2}=i_{2}$, etc., so that a nonzero contribution comes only from the terms with $\sigma=1$ and $j_{a}=i_{a}$ for all $a=1, \ldots, k$. Applying Lemma 4.7 and formulas (4.5) and (4.8) again we find that the contributions of the quantum minors are found by

$$
\operatorname{qdet} L^{-}(z) \mapsto l_{1}^{-}\left(z q^{-2 n+2}\right) \ldots l_{n}^{-}(z)
$$

and

$$
L^{-}(z) \underset{1 \ldots \hat{i} \ldots n}{1 \ldots \hat{i} \ldots n} \mapsto l_{1}^{-}\left(z q^{-2 n+4}\right) \ldots l_{i-1}^{-}\left(z q^{-2 n+2 i}\right) l_{i+1}^{-}\left(z q^{-2 n+2 i+2}\right) \ldots l_{n}^{-}(z) .
$$

This completes the calculation of the Harish-Chandra image of $\ell_{k}(z)$.

Consider the restriction of the map (6.1) to the subalgebra $\mathrm{Y}_{q}\left(\mathfrak{g l}_{n}\right)$ of $\widetilde{\mathrm{U}}_{q}\left(\widehat{\mathfrak{g l}}_{n}\right)_{\text {cri }}$. As in Sec. 3, we impose the conditions $l_{i i}^{+}[0]=1$ for all $i$ so that

$$
\chi: \mathrm{Y}_{q}\left(\mathfrak{g l}_{n}\right) \rightarrow \Pi_{q}^{+}(n),
$$

where $\Pi_{q}^{+}(n)$ is the subalgebra of $\Pi_{q}(n)$ generated by the variables $l_{i}^{+}[-r]$ with $i=1, \ldots, n$ and $r=0,1, \ldots$ subject to the relations $l_{i}^{+}[0]=1$ for all $i$. Recall the $q$-determinant calculated in Proposition 4.6. The following corollary essentially reproduces the $q$-deformed Miura transformation of [17]. 
Corollary 6.3. We have

$$
\chi: \operatorname{det}_{q}\left(\Pi+L^{+}(z) D \delta\right) \mapsto\left(1+\bar{\lambda}_{1}(z) \delta\right) \ldots\left(1+\bar{\lambda}_{n}(z) \delta\right)
$$

where $\bar{\lambda}_{i}(z)=q^{n-2 i+1} l_{i}^{+}(z)$.

Proof. This is immediate from Proposition 4.6 and Theorem 6.2.

In the remainder of this section we outline an alternative construction of the HarishChandra homomorphism for the quantum affine algebra $\mathrm{U}_{q}\left(\widehat{\mathfrak{g l}}_{n}\right)_{\text {cri }}$. The starting point is the version of the Poincaré-Birkhoff-Witt theorem for a different ordering on the generators as provided by Proposition 5.2. The arguments are essentially the same, with only minor changes in notation. As above, we define the projection $\theta^{\prime}: \mathrm{U}_{q}\left(\widehat{\mathfrak{g l}}_{n}\right)_{\text {cri }} \rightarrow \mathrm{U}^{0}$ in the same way. Proposition 6.1 holds in the same form but for the different Harish-Chandra homomorphism

$$
\chi^{\prime}: \mathrm{Z}_{q}\left(\widehat{\mathfrak{g l}}_{n}\right) \rightarrow \widetilde{\Pi}_{q}(n)
$$

defined as the restriction of the composition $\chi^{\prime}=\eta \circ \theta^{\prime}$. For $i=1, \ldots, n$ set

$$
\lambda_{i}^{\prime}(z)=q^{n-2 i+1} \frac{l_{i}^{+}(z) l_{i+1}^{-}\left(z q^{n-2 i}\right) \ldots l_{n}^{-}\left(z q^{-n+2}\right)}{l_{i}^{-}\left(z q^{n-2 i}\right) \ldots l_{n}^{-}\left(z q^{-n}\right)} .
$$

This is a Laurent series in $z$ whose coefficients are elements of the completed algebra $\widetilde{\Pi}_{q}(n)$.

Theorem 6.4. For each $k=1, \ldots, n$ the image of the series $\ell_{k}(z)$ under the HarishChandra homomorphism (6.5) is found by

$$
\chi^{\prime}: \ell_{k}(z) \mapsto \sum_{n \geqslant i_{1}>\cdots>i_{k} \geqslant 1} \lambda_{i_{1}}^{\prime}(z) \lambda_{i_{2}}^{\prime}\left(z q^{-2}\right) \ldots \lambda_{i_{k}}^{\prime}\left(z q^{-2 k+2}\right)
$$

Proof. The starting point is formula (4.2) and the argument is quite similar to the proof of Theorem 6.2.

\section{Eigenvalues in Wakimoto modules}

Our goal in this section is to relate the image of the series $\ell_{k}(z)$ under the Harish-Chandra homomorphism provided by Theorem 6.2 with their eigenvalues in the $q$-deformed Wakimoto modules constructed by Awata, Odake and Shiraishi [1]. Equivalently, due to the work of Frenkel and Reshetikhin [17], these eigenvalues can be interpreted as elements of the $q$-deformed classical $\mathcal{W}$-algebra $\mathcal{W}_{q}\left(\mathfrak{g l}_{n}\right)$. They were associated in [17] to the series $\ell_{V_{k}}(z)$ corresponding to fundamental representations $V_{k}$ of the quantum affine algebra $\mathrm{U}_{q}\left(\widehat{\mathfrak{s l}}_{n}\right)$. 
To establish the relationship, we consider the Wakimoto modules at the critical level over $\mathrm{U}_{q}\left(\widehat{\mathfrak{g l}}_{n}\right)$. The coefficients of the series $\ell_{k}(z)$ act as multiplications by scalars in the irreducible modules. We will show that these scalars can be found from Theorem 6.2 by an appropriate identification of the parameters of the Wakimoto modules with elements of $\Pi_{q}(n)$.

The free field realization of [1] is given in terms of Drinfeld's "new realization" [11] of the quantum affine algebra. Following [17], we will use the Ding-Frenkel isomorphism [10] to get the formulas for the action of the generators of $\mathrm{U}_{q}\left(\widehat{\mathfrak{g l}}_{n}\right)$ in the Wakimoto modules in terms of the $R L L$ presentation. Introduce the series $e_{i j}^{ \pm}(u), f_{i j}^{ \pm}(u)$ and $k_{i}^{ \pm}(u)$ which are uniquely determined by the Gauss decompositions of the respective matrices $L^{ \pm}(u)$ :

$$
\begin{aligned}
& L^{ \pm}(u)= \\
& {\left[\begin{array}{cccc}
1 & 0 & \ldots & 0 \\
e_{21}^{ \pm}(u) & \ddots & \ddots & \vdots \\
\vdots & \ddots & \ddots & 0 \\
e_{n 1}^{ \pm}(u) & \ldots & e_{n n-1}^{ \pm}(u) & 1
\end{array}\right]\left[\begin{array}{cccc}
k_{1}^{ \pm}(u) & 0 & \ldots & 0 \\
0 & \ddots & \ddots & \vdots \\
\vdots & \ddots & \ddots & 0 \\
0 & \ldots & 0 & k_{n}^{ \pm}(u)
\end{array}\right]\left[\begin{array}{cccc}
1 & f_{12}^{ \pm}(u) & \ldots & f_{1 n}^{ \pm}(u) \\
0 & \ddots & \ddots & \vdots \\
\vdots & \ddots & \ddots & f_{n-1 n}^{ \pm}(u) \\
0 & \ldots & 0 & 1
\end{array}\right]}
\end{aligned}
$$

We will need the following quantum minor expressions for these series. Their Yangian counterparts go back to [11] and detailed arguments were given in [4; see also [23, Sec. 1.11]. The quantum affine algebra case is quite similar so we only sketch the main steps of the proof.

Lemma 7.1. We have

$$
k_{i}^{ \pm}(u)=L^{ \pm}\left(q^{2 i-2} u\right)_{1 \cdots i}^{1 \cdots i}\left[L^{ \pm}\left(q^{2 i-2} u\right)_{1 \cdots i-1}^{1 \cdots i-1}\right]^{-1}
$$

for $i=1, \ldots, n$ and

$$
\begin{aligned}
& e_{j i}^{ \pm}(u)=L^{ \pm}\left(q^{2 i-2} u\right)_{1 \cdots i}^{1 \cdots i-1 j}\left[L^{ \pm}\left(q^{2 i-2} u\right)_{1 \cdots i}^{1 \cdots i}\right]^{-1} \\
& =q^{-1}\left[L^{ \pm}\left(q^{2 i} u\right)_{1 \cdots i}^{1 \cdots i}\right]^{-1} L^{ \pm}\left(q^{2 i} u\right)_{1 \cdots i}^{1 \cdots i-1 j}, \\
& f_{i j}^{ \pm}(u)=\left[L^{ \pm}\left(q^{2 i-2} u\right)_{1 \cdots i}^{1 \cdots i}\right]^{-1} L^{ \pm}\left(q^{2 i-2} u\right)_{1 \cdots i-1 j}^{1 \cdots i}=q L^{ \pm}\left(q^{2 i} u\right)_{1 \cdots i-1 j}^{1 \cdots i}\left[L^{ \pm}\left(q^{2 i} u\right)_{1 \cdots i}^{1 \cdots i}\right]^{-1}
\end{aligned}
$$

for $1 \leqslant i<j \leqslant n$.

Proof. The arguments for the matrices $L^{+}(u)$ and $L^{-}(u)$ are the same so we will work with $L^{+}(u)$ and use the notation $\mathrm{Y}_{q}\left(\mathfrak{g l}_{n}\right)$ for the subalgebra of the quantum affine algebra generated by the coefficients of all series $l_{i j}^{+}(u)$. We will also use the algebra $Y_{q^{-1}}\left(\mathfrak{g l}_{n}\right)$ and denote its generator matrix by $\widetilde{L}^{+}(u)=\left[\widetilde{l}_{i j}^{+}(u)\right]$. Due to the property

$$
R_{21}(v, u)=-\left.R(u, v)\right|_{q \mapsto q^{-1}}
$$


of the $R$-matrix (2.1), the mapping

$$
\omega_{n}: L^{+}(u) \mapsto \widetilde{L}^{+}(u)^{-1}
$$

defines a homomorphism $\omega_{n}: \mathrm{Y}_{q}\left(\mathfrak{g l}_{n}\right) \rightarrow \mathrm{Y}_{q^{-1}}\left(\mathfrak{g l}_{n}\right)$. For any $m \geqslant 0$ consider another homomorphism

$$
\jmath_{m}: \mathrm{Y}_{q^{-1}}\left(\mathfrak{g l}_{n}\right) \rightarrow \mathrm{Y}_{q^{-1}}\left(\mathfrak{g l}_{m+n}\right)
$$

which takes the coefficients of the series $\widetilde{l}_{i j}^{+}(u)$ to the respective coefficients of the series $\widetilde{l}_{m+i m+j}^{+}(u)$. Consider the composition $\phi_{m}=\omega_{m+n}^{-1} \circ \jmath_{m} \circ \omega_{n}$ which is an algebra homomorphism

$$
\phi_{m}: \mathrm{Y}_{q}\left(\mathfrak{g l}_{n}\right) \rightarrow \mathrm{Y}_{q}\left(\mathfrak{g l}_{m+n}\right)
$$

By [22, Lemma 3.7]

$$
\phi_{m}: l_{i j}^{+}(u) \mapsto\left[L^{+}\left(q^{2 m} u\right)_{1 \cdots m}^{1 \cdots m}\right]^{-1} L^{+}\left(q^{2 m} u\right)_{1 \cdots m m+j}^{1 \cdots m m+i} .
$$

Now apply [23, Lemmas 1.11.2 and 1.11.5] to the algebra $\mathrm{Y}_{q}\left(\mathfrak{g l}_{n}\right)$ to get

$$
\begin{aligned}
k_{i}^{+}(u) & =\phi_{i-1}\left(l_{11}^{+}(u)\right), \\
e_{j i}^{+}(u) & =\phi_{i-1}\left(l_{j-i+11}^{+}(u) l_{11}^{+}(u)^{-1}\right), \\
f_{i j}^{+}(u) & =\phi_{i-1}\left(l_{11}^{+}(u)^{-1} l_{1 j-i+1}^{+}(u)\right) .
\end{aligned}
$$

Together with (7.4) this proves the formula for $k_{i}^{+}(u)$ and the first expressions for $e_{j i}^{+}(u)$ and $f_{i j}^{+}(u)$. To prove the second expressions, use the relations

$$
l_{j-i+11}^{+}(u) l_{11}^{+}(u)^{-1}=q^{-1} l_{11}^{+}\left(q^{2} u\right)^{-1} l_{j-i+11}^{+}\left(q^{2} u\right)
$$

and

$$
l_{11}^{+}(u)^{-1} l_{1 j-i+1}^{+}(u)=q l_{1 j-i+1}^{+}\left(q^{2} u\right) l_{11}^{+}\left(q^{2} u\right)^{-1}
$$

in $\mathrm{Y}_{q}\left(\mathfrak{g l}_{n}\right)$ implied by (2.7) (or (5.5)) and apply (7.4) again.

Corollary 7.2. We have the expansions for the quantum determinants

$$
\text { qdet } L^{ \pm}(z)=k_{1}^{ \pm}(z) k_{2}^{ \pm}\left(z q^{-2}\right) \ldots k_{n}^{ \pm}\left(z q^{-2 n+2}\right) .
$$

Proof. This is immediate from Lemma 7.1 .

We adopt the notation $\psi_{ \pm}^{i}(z)$ and $E^{ \pm, i}(z)$ of [1] for the series of Drinfeld generators of $\mathrm{U}_{q}\left(\widehat{\mathfrak{s l}}_{n}\right)$ and identify them with the respective elements of $\mathrm{U}_{q}\left(\widehat{\mathfrak{g l}}_{n}\right)$ by using the isomorphism of [10] in the normalization of [16]. The formulas are given in [16] only for the zero level

\footnotetext{
${ }^{3}$ The generator matrices $T(u)$ and $\bar{T}(u)$ of 22 correspond to $L^{-}(u)$ and $L^{+}(u)$, respectively.
} 
case, but they can be easily modified to include the central element $q^{c}$ as in [10]. For $i=1, \ldots, n-1$ we have

$$
\begin{aligned}
\psi_{ \pm}^{i}(z) & =k_{i+1}^{\mp}\left(z q^{-i}\right) k_{i}^{\mp}\left(z q^{-i}\right)^{-1} \\
E^{+, i}(z) & =\frac{e_{i+1 i}^{+}\left(z q^{\frac{c}{2}-i}\right)-e_{i+1 i}^{-}\left(z q^{-\frac{c}{2}-i}\right)}{\left(q-q^{-1}\right) z} \\
E^{-, i}(z) & =\frac{f_{i+1}^{+}\left(z q^{-\frac{c}{2}-i}\right)-f_{i i+1}^{-}\left(z q^{\frac{c}{2}-i}\right)}{\left(q-q^{-1}\right) z} .
\end{aligned}
$$

In fact, these relations were established in [10] for the respective elements of the algebra $\overline{\mathrm{U}}_{q}\left(\widehat{\mathfrak{g l}}_{n}\right)$ which we defined in Sec. 5. However, the map (5.7) connecting the two algebras only results in the multiplication of the series $k_{i}^{+}(z)$ by a scalar series with coefficients in the Heisenberg algebra $\mathcal{H}_{q}(n)$ and in the multiplication of the series $k_{i}^{-}(z)$ by a different scalar series. The series $e_{i j}^{ \pm}(u)$ and $f_{i j}^{ \pm}(u)$ are not affected and hence the above relations apply to the algebra $\mathrm{U}_{q}\left(\widehat{\mathfrak{g l}}_{n}\right)$ in the same form.

The $q$-deformed Wakimoto modules over $\mathrm{U}_{q}\left(\widehat{\mathfrak{s l}}_{n}\right)$ are realized in the boson Fock space by an explicit action of the series $\psi_{ \pm}^{i}(z)$ and $E^{ \pm, i}(z)$ described in [1]. We recall this construction assuming that the level is critical. That is, we take $k=-n$ in the notation of [1] and for all $1 \leqslant i<j \leqslant n$ consider free boson fields

$$
\begin{aligned}
& b^{i j}(z)=-\sum_{r \neq 0} \frac{b_{r}^{i j}}{[r]} z^{-r}+\frac{q-q^{-1}}{2 \log q} b_{0}^{i j} \log z+Q_{b}^{i j}, \\
& c^{i j}(z)=-\sum_{r \neq 0} \frac{c_{r}^{i j}}{[r]} z^{-r}+\frac{q-q^{-1}}{2 \log q} c_{0}^{i j} \log z+Q_{c}^{i j},
\end{aligned}
$$

and

$$
\begin{aligned}
& b_{ \pm}^{i j}(z)= \pm\left(q-q^{-1}\right)\left(\frac{b_{0}^{i j}}{2}+\sum_{ \pm r>0} b_{r}^{i j} z^{-r}\right), \\
& c_{ \pm}^{i j}(z)= \pm\left(q-q^{-1}\right)\left(\frac{c_{0}^{i j}}{2}+\sum_{ \pm r>0} c_{r}^{i j} z^{-r}\right),
\end{aligned}
$$

where

$$
[r]=\frac{q^{r}-q^{-r}}{q-q^{-1}}
$$

and the coefficients satisfy the relations

$$
\begin{array}{lll}
{\left[b_{r}^{i j}, b_{s}^{k l}\right]=-\frac{[r]^{2}}{r} \delta_{i k} \delta_{j l} \delta_{r,-s},} & {\left[b_{0}^{i j}, Q_{b}^{k l}\right]=-\frac{2 \log q}{q-q^{-1}} \delta_{i k} \delta_{j l},} \\
{\left[c_{r}^{i j}, c_{s}^{k l}\right]=\frac{[r]^{2}}{r} \delta_{i k} \delta_{j l} \delta_{r,-s},} & {\left[c_{0}^{i j}, Q_{c}^{k l}\right]=\frac{2 \log q}{q-q^{-1}} \delta_{i k} \delta_{j l},}
\end{array}
$$


all other pairs of coefficients commute. The quantum Heisenberg algebra $\mathcal{A}_{q}(n)$ is generated by the elements $e^{ \pm Q_{b}^{i j}}, e^{ \pm Q_{c}^{i j}}, e^{ \pm\left(q-q^{-1}\right) b_{0}^{i j} / 2}, e^{ \pm\left(q-q^{-1}\right) c_{0}^{i j} / 2}$ and $b_{r}^{i j}, c_{r}^{i j}$ with $r \neq 0$. The defining relations are those implied by the above commutations relations for the coefficients of the free boson fields. The Fock representation $F_{q}(n)$ of $\mathcal{A}_{q}(n)$ is generated by the vacuum vector $|0\rangle$ such that

$$
b_{r}^{i j}|0\rangle=c_{r}^{i j}|0\rangle=0 \quad \text { for all } i<j \text { and } r \geqslant 0
$$

so that, in particular,

$$
e^{ \pm\left(q-q^{-1}\right) b_{0}^{i j} / 2}|0\rangle=e^{ \pm\left(q-q^{-1}\right) c_{0}^{i j} / 2}|0\rangle=|0\rangle .
$$

The generators $a_{r}^{i}$ with $i=1, \ldots, n-1$ and $r \in \mathbb{Z}$ used in the free field realization of [1] pairwise commute at the critical level. Therefore, they may be regarded as numerical parameters of the $q$-deformed Wakimoto modules and we must have $a_{r}^{i}=0$ for $r \geqslant 0$. For the use in the formulas below we set

$$
a_{ \pm}^{i}(z)= \pm\left(q-q^{-1}\right)\left(\frac{1}{2} a_{0}^{i}+\sum_{ \pm r>0} a_{r}^{i} z^{-r}\right),
$$

as in [1] and we have $a_{+}^{i}(z)=0$ for all $i$. The ( $q$-deformed) Wakimoto module over $\mathrm{U}_{q}\left(\widehat{\mathfrak{s l}}_{n}\right)$ at the critical level is defined by the action of the Drinfeld generators in the space $F_{q}(n)$. For the action of the coefficients of the series $\psi_{ \pm}^{i}(z)$ we have

$$
\begin{aligned}
\psi_{ \pm}^{i}\left(z q^{\mp n / 2}\right)=\exp & \left(\sum_{j=1}^{i}\left(b_{ \pm}^{j i+1}\left(z q^{ \pm(j-n-1)}\right)-b_{ \pm}^{j i}\left(z q^{ \pm(j-n)}\right)\right)\right. \\
& \left.+a_{ \pm}^{i}(z)+\sum_{j=i+1}^{n}\left(b_{ \pm}^{i j}\left(z q^{ \pm(j-n)}\right)-b_{ \pm}^{i+1 j}\left(z q^{ \pm(j-n-1)}\right)\right)\right),
\end{aligned}
$$

where $b_{ \pm}^{i i}(z)=c_{ \pm}^{i i}(z)=0$. The coefficients of $E^{+, i}(z)$ act by

$$
\begin{aligned}
E^{+, i}(z) & =\frac{1}{\left(q-q^{-1}\right) z} \sum_{j=1}^{i}: \exp \left((b+c)^{j i}\left(z q^{j-1}\right)\right) \\
& \times\left(\exp \left(b_{-}^{j i+1}\left(z q^{j-1}\right)-(b+c)^{j i+1}\left(z q^{j-2}\right)\right)-\exp \left(b_{+}^{j i+1}\left(z q^{j-1}\right)-(b+c)^{j i+1}\left(z q^{j}\right)\right)\right) \\
& \times \exp \left(\sum_{l=1}^{j-1}\left(b_{+}^{l i+1}\left(z q^{l-1}\right)-b_{+}^{l i}\left(z q^{l}\right)\right)\right):
\end{aligned}
$$

where we have used the notation $(b+c)^{i j}(z)=b^{i j}(z)+c^{i j}(z)$ and set $b^{i i}(z)=c^{i i}(z)=0$. The colons indicate normal ordering so that the coefficients $b_{r}^{i j}$ with $r<0$ or $\exp Q_{b}^{i j}$ should be placed to the left of the coefficients $b_{r}^{i j}$ with $r \geqslant 0$. The same rule applies to the coefficients 
of $c^{i j}(z)$. We will not reproduce the formulas for the action of $E^{-, i}(z)$ as they are given by longer expressions and will not be used; see [1, (3.7)]. Our notation is the same as in [1] for $b_{r}^{i j}$ and $c_{r}^{i j}$, whereas $Q_{b}^{i j}=\hat{q}_{b}^{i j}$ and $Q_{c}^{i j}=\hat{q}_{c}^{i j}$.

By Lemma 4.3, the coefficients of the quantum determinants are central in the algebra $\mathrm{U}_{q}\left(\widehat{\mathfrak{g l}}_{n}\right)$ at the critical level. Therefore, the irreducible Wakimoto modules can be extended to $\mathrm{U}_{q}\left(\widehat{\mathfrak{g l}}_{n}\right)_{\text {cri }}$ by specifying the eigenvalues $K^{ \pm}(z)$ of qdet $L^{ \pm}(z)$. By Corollary 7.2 , this gives the conditions

$$
k_{1}^{ \pm}(z) k_{2}^{ \pm}\left(z q^{-2}\right) \ldots k_{n}^{ \pm}\left(z q^{-2 n+2}\right) \mapsto K^{ \pm}(z),
$$

where $K^{+}(z)$ and $K^{-}(z)$ are power series in $z$ and $z^{-1}$, respectively. Hence, relations (7.5) and (7.9) allow us to define the action of the coefficients of all series $k_{i}^{ \pm}(z)$ on the Fock space.

For any $X \in \mathrm{U}_{q}\left(\widehat{\mathfrak{g l}}_{n}\right)_{\text {cri }}$ we will write $\langle 0|X| 0\rangle$ to denote the coefficient of $|0\rangle$ in the expansion of $X|0\rangle$ along the basis of the Fock space 4 More generally, a relation of the form $\langle 0| X=d\langle 0|$ for a constant $d$ will be understood in the sense that $\langle 0|X Y| 0\rangle=d\langle 0|Y| 0\rangle$ for any element $Y \in \mathrm{U}_{q}\left(\widehat{\mathfrak{g l}}_{n}\right)_{\text {cri }}$. Using this notation we can parameterize the corresponding modules over $\mathrm{U}_{q}\left(\widehat{\mathfrak{g l}}_{n}\right)_{\text {cri }}$ by the power series $\varkappa_{i}^{+}(z)$ and $\varkappa_{i}^{-}(z)$ in $z$ and $z^{-1}$, respectively, such that

$$
k_{i}^{-}(z)|0\rangle=\varkappa_{i}^{-}(z)|0\rangle \quad \text { and } \quad\langle 0| k_{i}^{+}(z)=\langle 0| \varkappa_{i}^{+}(z)
$$

for all $i=1, \ldots, n$ satisfying the relations

$$
\varkappa_{i+1}^{-}(z) \varkappa_{i}^{-}(z)^{-1}=\exp \left(a_{+}^{i}\left(z q^{\frac{n}{2}+i}\right)\right) \quad \text { and } \quad \varkappa_{i+1}^{+}(z) \varkappa_{i}^{+}(z)^{-1}=\exp \left(a_{-}^{i}\left(z q^{-\frac{n}{2}+i}\right)\right)
$$

for $i=1, \ldots, n-1$. Since $a_{+}^{i}(z)=0$, the series $\varkappa_{i}^{-}(z)$ is the same for each $i$ and we denote it by $\varkappa^{-}(z)$.

The following theorem is essentially due to [17] subject to the identification of $\ell_{k}(z)$ with the series $\ell_{V_{k}}(z)$ corresponding to the fundamental representation $V_{k}$ of $\mathrm{U}_{q}\left(\widehat{\mathfrak{s l}}_{n}\right)$, although the arguments were only outlined there. The eigenvalues of $\ell_{V_{k}}(z)$ were interpreted in [17] as generators of the $q$-deformed classical $\mathcal{W}$-algebras and the Poisson brackets between the generators were explicitly calculated.

Theorem 7.3. Given an irreducible Wakimoto module over $\mathrm{U}_{q}\left(\widehat{\mathfrak{g l}}_{n}\right)_{\text {cri }}$ with the parameters $\varkappa_{i}^{+}(z)$ and $\varkappa^{-}(z)$, the eigenvalues of the coefficients of the series $\ell_{k}(z)$ in the module are found by

$$
\ell_{k}(z) \mapsto \sum_{1 \leqslant i_{1}<\cdots<i_{k} \leqslant n} \Lambda_{i_{1}}(z) \Lambda_{i_{2}}\left(z q^{-2}\right) \ldots \Lambda_{i_{k}}\left(z q^{-2 k+2}\right), \quad k=1, \ldots, n,
$$

where

$$
\Lambda_{i}(z)=q^{n-2 i+1} \varkappa_{i}^{+}(z) \varkappa^{-}\left(z q^{-n}\right)^{-1}, \quad i=1, \ldots, n .
$$

\footnotetext{
${ }^{4} \mathrm{An}$ equivalent interpretation would involve an inner product on the Fock space which we will not introduce.
} 
Proof. Any irreducible Wakimoto module coincides with the cyclic span over $\mathrm{U}_{q}\left(\widehat{\mathfrak{g l}}_{n}\right)_{\text {cri }}$ of the vacuum vector $|0\rangle$. Hence, the eigenvalues of the coefficients of the series $\ell_{k}(z)$ can be found by calculating the series $\left\langle 0\left|\ell_{k}(z)\right| 0\right\rangle$.

We find from (7.10) that $E^{+, i}(z)|0\rangle$ is a power series in $z$ for all $i$. Therefore, (7.6) implies $e_{i+1 i}^{-}(z)|0\rangle=0$ for $i=1, \ldots, n-1$. Note the following relations for the action of the generators $l_{i j}^{-}[0]$ on the vacuum vector:

$$
l_{i+1 i}^{-}[0]|0\rangle=0 \quad \text { and } \quad l_{i i}^{-}[0]|0\rangle=\varkappa^{-}[0]|0\rangle,
$$

where $\varkappa^{-}[0]$ denotes the constant term of the series $\varkappa^{-}(z)$. Indeed, using (7.1) and (7.11), we find that $L^{-}(z)_{1 \cdots i}^{1 \cdots i}|0\rangle$ is a scalar power series in $z^{-1}$. Expanding the quantum minor by (4.5) and taking the constant term we find that

$$
\sum_{\sigma \in \mathfrak{S}_{i}}(-q)^{-l(\sigma)} l_{\sigma(1) 1}^{-}[0] \cdots l_{\sigma(i) i}^{-}[0]|0\rangle
$$

is a scalar multiple of the vacuum vector $|0\rangle$. However, $l_{j i}^{-}[0]=0$ for $j<i$ by (2.5) so that the only nonzero term in (7.13) corresponds to the identity permutation $\sigma$. Therefore, taking the constant terms in (17.1) and (7.11) we derive the second relation in (7.12). Now use the relation $L^{-}(z)_{1 \cdots i}^{1 \cdots i-1 i+1}|0\rangle=0$ implied by (7.2). Exactly as above, we get

$$
l_{11}^{-}[0] \cdots l_{i-1 i-1}^{-}[0] l_{i+1 i}^{-}[0]|0\rangle=0
$$

which gives the first relation in (17.12) by (5.2). As a next step, we will derive the relations

$$
e_{j i}^{-}(z)|0\rangle=0 \quad \text { for all } j>i \text {. }
$$

It follows from (2.7) that

$$
\left[l_{i j}^{-}[0], l_{j m}^{-}(z)\right]=\left(q-q^{-1}\right) l_{i m}^{-}(z) l_{j j}^{-}[0] \quad \text { for } \quad i>j>m
$$

and

$$
\left[l_{i j}^{-}[0], l_{k m}^{-}(z)\right]=0 \quad \text { for } \quad i, j>k, m \text {. }
$$

Hence, (17.2) gives

$$
\left[l_{j+1 j}^{-}[0], e_{j i}^{-}(z)\right]=\left(q-q^{-1}\right) e_{j+1 i}^{-}(z) l_{j j}^{-}[0]
$$

and (7.15) follows by induction from (7.12).

Thus, we may conclude that

$$
L^{-}(z)_{1 \cdots i}^{1 \cdots i-1 j}|0\rangle=0 \quad \text { for all } j>i .
$$

Expanding the quantum minor with the use of (4.5) and (4.8) we get

$$
\sum_{\sigma}(-q)^{l(\sigma)} l_{\sigma(j) i}^{-}(z) l_{\sigma(i-1) i-1}^{-}\left(q^{-2} z\right) \cdots l_{\sigma(1) 1}^{-}\left(q^{-2 i+2} z\right)|0\rangle=0,
$$


where the sum is taken over permutations $\sigma$ of the set $\{1, \ldots, i-1, j\}$. Together with the property that

$$
L^{-}(z)_{1 \cdots i}^{1 \cdots i}|0\rangle=\sum_{\sigma \in \mathfrak{S}_{i}}(-q)^{l(\sigma)} l_{\sigma(i) i}^{-}(z) \cdots l_{\sigma(1) 1}^{-}\left(q^{-2 i+2} z\right)|0\rangle
$$

is a scalar power series in $z^{-1}$, these relations imply that

$$
l_{j i}^{-}(z)|0\rangle=0 \quad \text { for all } j>i
$$

and $l_{i i}^{-}(z)|0\rangle$ is a scalar power series in $z^{-1}$ by an obvious induction. Moreover, it follows from (7.1) and (7.11) that

$$
l_{i i}^{-}(z)|0\rangle=\varkappa^{-}(z)|0\rangle \quad \text { for } i=1, \ldots, n .
$$

Now we will apply similar arguments to derive that

$$
\langle 0| l_{j i}^{+}(z)=0 \quad \text { for all } j>i \quad \text { and } \quad\langle 0| l_{i i}^{+}(z)=\langle 0| \varkappa_{i}^{+}(z) \text { for } i=1, \ldots, n .
$$

The first step is to observe that $\langle 0| z E^{+, i}(z)$ is a power series in $z^{-1}$. Indeed, this follows from (7.10) with the use of the relations

$$
\langle 0| b_{r}^{i j}=\langle 0| c_{r}^{i j}=0 \quad \text { for all } r \geqslant 0 .
$$

One additional step is to use the commutation relations (17.7) and (17.8) which imply

$$
\exp Q_{b}^{i j} \cdot z^{\frac{q-q^{-1}}{2 \log q} b_{0}^{i j}}=z^{\frac{q-q^{-1}}{2 \log q} b_{0}^{i j}} \cdot \exp Q_{b}^{i j} \cdot z
$$

and

$$
\exp Q_{c}^{i j} \cdot z^{\frac{q-q^{-1}}{2 \log q} c_{0}^{i j}}=z^{\frac{q-q^{-1}}{2 \log q} c_{0}^{i j}} \cdot \exp Q_{c}^{i j} \cdot z^{-1} .
$$

Although extra powers of $z$ occur as a result of swapping the coefficients, these powers arising from the coefficients of the series $b^{i j}(z)$ and $c^{i j}(z)$ cancel each other. Thus, using (7.6) and noting that the constant term of $e_{i+1 i}^{+}(z)$ is zero, we come to the relation $\langle 0| e_{i+1 i}^{+}(z)=0$ for $i=1, \ldots, n-1$. The rest of the arguments is essentially the same with some obvious adjustments. In particular, to evaluate the constant term of the power series $\langle 0| L^{+}(z)_{1 \cdots i}^{1 \cdots i}$ we use the expansion

$$
\langle 0| \sum_{\sigma \in \mathfrak{S}_{i}}(-q)^{l(\sigma)} l_{\sigma(i) i}^{+}[0] \cdots l_{\sigma(1) 1}^{+}[0]
$$

instead of (7.13) and note that $l_{j i}^{+}[0]=0$ for $j>i$. Together with the corresponding counterpart of (7.14) this implies

$$
\langle 0| l_{i+1 i}^{+}[0]=0 \quad \text { and } \quad\langle 0| l_{i i}^{+}[0]=\langle 0| \varkappa_{i}^{+}[0],
$$


where $\varkappa_{i}^{+}[0]$ denotes the constant term of the series $\varkappa_{i}^{+}(z)$. In the final part we use the relations

$$
\langle 0| \sum_{\sigma}(-q)^{-l(\sigma)} l_{\sigma(1) 1}^{+}(z) \ldots l_{\sigma(i-1) i-1}^{+}\left(q^{-2 i+4} z\right) l_{\sigma(j) i}^{+}\left(q^{-2 i+2} z\right)=0
$$

with the sum over permutations $\sigma$ of the set $\{1, \ldots, i-1, j\}$, and

$$
\langle 0| L^{+}(z)_{1 \cdots i}^{1 \cdots i}=\langle 0| \sum_{\sigma \in \mathfrak{S}_{i}}(-q)^{-l(\sigma)} l_{\sigma(1) 1}^{+}(z) \ldots l_{\sigma(i) i}^{+}\left(q^{-2 i+2} z\right)
$$

instead of (7.17) and (7.18).

Relations (7.19), (7.20) and (7.21) allow us to conclude that the eigenvalue $\left\langle 0\left|\ell_{k}(z)\right| 0\right\rangle$ coincides with the image of the series $\ell_{k}(z)$ under the Harish-Chandra homomorphism calculated in Theorem 6.2 for the specialization

$$
l_{i}^{+}(z)=\varkappa_{i}^{+}(z) \quad \text { and } \quad l_{i}^{-}(z)=\varkappa^{-}(z)
$$

for $i=1, \ldots, n$. Clearly, then $\lambda_{i}(z)$ specializes to $\Lambda_{i}(z)$ and the proof is complete.

Remark 7.4. The fact that the eigenvalues of the coefficients of $\ell_{k}(z)$ in the Wakimoto modules are consistent with the Harish-Chandra images provided by Theorem 6.2 relies on the properties (7.19), (7.20) and (7.21). It was essential for their derivation that the "zero mode matrices" $L^{+}[0]$ and $L^{-}[0]$ are upper and lower triangular, respectively. These properties do not hold for the presentation of the quantum affine algebra used in [17], where the triangularity of the zero mode matrices is opposite.

\section{References}

[1] H. Awata, S. Odake and J. Shiraishi, Free boson realization of $U_{q}\left(\widehat{\mathfrak{s l}}_{N}\right)$, Comm. Math. Phys. 162 (1994), 61-83.

[2] J. Beck, Convex bases of PBW type for quantum affine algebras, Comm. Math. Phys. 165 (1994), 193-199.

[3] J. Beck, Braid group action and quantum affine algebras, Comm. Math. Phys. 165 (1994), 555-568.

[4] J. Brundan and A. Kleshchev, Parabolic presentations of the Yangian $\mathrm{Y}\left(\mathfrak{g l}_{n}\right)$, Commun. Math. Phys. 254 (2005), 191-220.

[5] I. V. Cherednik, A new interpretation of Gelfand-Tzetlin bases, Duke Math. J. 54 (1987), 563-577. 
[6] A. Chervov, G. Falqui, V. Rubtsov and A. Silantyev, Algebraic properties of Manin matrices II: q-analogues and integrable systems, Adv. in Appl. Math. 60 (2014), 25-89.

[7] A. V. Chervov and A. I. Molev, On higher order Sugawara operators, Int. Math. Res. Not. (2009), no. 9, 1612-1635.

[8] A. Chervov and D. Talalaev, Quantum spectral curves, quantum integrable systems and the geometric Langlands correspondence, arXiv:hep-th/0604128.

[9] J. Ding and P. Etingof, The center of a quantum affine algebra at the critical level, Math. Res. Lett. 1 (1994), 469-480.

[10] J. Ding and I. B. Frenkel, Isomorphism of two realizations of quantum affine algebra $U_{q}(\widehat{\mathfrak{g l}}(n))$, Comm. Math. Phys. 156 (1993), 277-300.

[11] V. G. Drinfeld, A new realization of Yangians and quantized affine algebras, Soviet Math. Dokl. 36 (1988), 212-216.

[12] P. Etingof and D. Kazhdan, Quantization of Lie bialgebras. V. Quantum vertex operator algebras, Selecta Math. (N.S.) 6 (2000), 105-130.

[13] B. Feigin, E. Frenkel and N. Reshetikhin, Gaudin model, Bethe ansatz and critical level, Comm. Math. Phys. 166 (1994), 27-62.

[14] B. Feigin, E. Frenkel and V. Toledano Laredo, Gaudin models with irregular singularities, Adv. Math. 223 (2010), 873-948.

[15] E. Frenkel, Langlands correspondence for loop groups, Cambridge Studies in Advanced Mathematics, 103. Cambridge University Press, Cambridge, 2007.

[16] E. Frenkel and E. Mukhin, The Hopf algebra Rep $U_{q} \widehat{\mathfrak{g l}}_{\infty}$, Selecta Math. 8 (2002), 537-635.

[17] E. Frenkel and N. Reshetikhin, Quantum affine algebras and deformations of Virasoro and $\mathcal{W}$-algebras, Comm. Math. Phys. 178 (1996), 237-264.

[18] E. Frenkel and N. Reshetikhin, The q-characters of representations of quantum affine algebras and deformations of $\mathcal{W}$-algebras, Contemp. Math. 248 (1999), 163-205.

[19] I. B. Frenkel and N. Jing, Vertex representations of quantum affine algebras, Proc. Nat. Acad. Sci. U.S.A. 85 (1988), 9373-9377.

[20] I. B. Frenkel and N. Yu. Reshetikhin, Quantum affine algebras and holonomic difference equations, Comm. Math. Phys. 146 (1992), 1-60. 
[21] L. Gow and A. Molev, Representations of twisted $q$-Yangians, Selecta Math., New Series 16 (2010), 439-499.

[22] M. J. Hopkins and A. I. Molev, A q-analogue of the centralizer construction and skew representations of the quantum affine algebra, Symmetry, Integrability and Geometry: Methods and Applications 2 (2006), paper 092, 29 pp.

[23] A. Molev, Yangians and classical Lie algebras, Mathematical Surveys and Monographs, 143. American Mathematical Society, Providence, RI, 2007.

[24] A. I. Molev, Feigin-Frenkel center in types B, C and D, Invent. Math. 191 (2013), 1-34.

[25] A. I. Molev and E. E. Mukhin, Yangian characters and classical $\mathcal{W}$-algebras, in "Conformal field theory, automorphic forms and related topics" (W. Kohnen, R. Weissauer, Eds), Springer, 2014, pp. 287-334.

[26] A. I. Molev and E. Ragoucy, The MacMahon Master Theorem for right quantum superalgebras and higher Sugawara operators for $\widehat{\mathfrak{g l}}_{m \mid n}$, Moscow Math. J. 14 (2014), 83-119.

[27] N. Yu. Reshetikhin and M. A. Semenov-Tian-Shansky, Central extensions of quantum current groups, Lett. Math. Phys. 19 (1990), 133-142.

[28] L. G. Rybnikov, The shift of invariants method and the Gaudin model, Funct. Anal. Appl. 40 (2006), 188-199. 
L.F. \& E.R.:

Laboratoire de Physique Théorique LAPTh, CNRS and Université de Savoie BP 110, 74941 Annecy-le-Vieux Cedex, France

luc.frappat@lapth.cnrs.fr

eric.ragoucy@lapth.cnrs.fr

N.J.:

School of Mathematical Sciences

South China University of Technology

Guangzhou, Guangdong 510640, China

and

Department of Mathematics

North Carolina State University, Raleigh, NC 27695, USA

jing@math.ncsu.edu

A.M.:

School of Mathematics and Statistics

University of Sydney, NSW 2006, Australia

alexander.molev@sydney.edu.au 